\title{
Qualitative analysis of a forced nonsmooth oscillator with contact and friction
}

\author{
Alain Léger · Elaine Pratt \\ A. Léger \\ Laboratoire de Mécanique et d'Acoustique, CNRS, \\ 31, chemin Joseph Aiguier, 13402 Marseille Cedex 20, France \\ E. Pratt \\ Aix-Marseille Université, Centre de Mathématiques et \\ d'Informatique, 39, rue F. Joliot Curie, \\ 13453 Marseille Cedex 13, France \\ e-mail: pratt@lma.cnrs-mrs.fr
}

\begin{abstract}
We study the qualitative dynamics of a simple mass-spring system involving non regularized unilateral contact and Coulomb friction and submitted to an oscillating external force. The period-amplitude plane of the excitation appears to be essentially divided into two ranges of sliding solutions. At each point of the lower range there exist infinitely many equilibrium points and all the trajectories go to equilibrium in finite time. In the upper range, there no longer exist equilibria. Different kinds of periodic solutions are shown to exist in different zones and the transitions between these zones are explicitly computed. The upper boundary of this range, where the mass looses contact, is also computed and special attention is paid to the dependence of this upper boundary with respect to the period of the excitation.
\end{abstract}

Keywords Coulomb friction · Mass-spring systems . Nonsmooth dynamics $\cdot$ Stability $\cdot$ Unilateral contact

\section{Introduction}

The present work is in the same vein as previous papers dealing with the stability of discrete mechanical systems involving nonsmooth unilateral contact and friction conditions. Bearing in mind that due to the dissipative character of the friction law and the nonsmooth character of both the contact and the friction condition, classical stability analysis is to be ruled out, our first option was to observe the trajectories starting from initial data close to equilibrium points to obtain stability properties. Having determined the set of equilibrium states of a simple model with unilateral contact and Coulomb friction their stability was initially explored by numerical experiments [5]. Then we came back to the idea of using analytical estimates of the evolution in time of the distance between a given equilibrium state and any trajectory starting from initial data sufficiently close to the equilibrium [3]. It was however observed that although such an analysis took very carefully into account the contact and friction constraints along the trajectory, it was not really satisfactory from the point of view of stability because of the specificity of the friction law. In fact, due to the graph of the Coulomb friction law, starting from a given equilibrium state and modifying the external forces induces a change in the reaction but may produce no change in the position nor in the velocity of the particles. This suggested a new notion of stability first given in [2]. The new notion was analyzed at length, and led to a conjecture, partially proved in [13].

The present work is formally very close to usual investigations of the behaviour of classical dynamical systems with respect to the parameters of the excitation. However we are not concerned for the time being by stability notions but essentially by some qualitative properties of the trajectories. Only the fact that some trajectories always lead to a stationnary solution can be seen as a contribution to a stability analysis. We focus on the case of a very simple mass-spring system already used in several papers (e.g. [5] or [3]), but here the system is submitted to 
an oscillating force. A rectangular wave excitation is chosen in order to show as explicitly as possible the complexity of the dynamics. Fundamental differences with respect to smooth dynamical systems are obtained in the period-amplitude plane of the excitation, such as the persistence of infinitely many equilibrium states while the right-hand side is oscillating.

The main steps of the paper are outlined as follows.

Section 2 summarizes previous results. In order to have a self-sufficient paper, it seemed necessary to describe the simple mass-spring system we are dealing with together with the basic equations of its dynamics, then to recall without proof some results concerning its qualitative dynamics, and finally to give the set of its equilibria under constant loading.

Section 3 gives the existence results of equilibrium points under an oscillating force, so that a first part of the classical period-amplitude plane is determined.

Section 4 is the main part of the work. In the range where equilibrium states no longer exist we study the existence and the characteristics of periodic solutions. We show that this range is divided into several zones between which the transitions correspond either to the value of the period, to the shape of the trajectory, or to the synchronization of the answer with the excitation.

Section 5 studies the upper boundary of these zones in the period-amplitude plane, which means that we explore the set of periodic solutions up to the occurence of an episode of motion where contact is lost. We then conclude by listing some remaining problems and in particular by observing that taking impacts into account will involve the nonsmoothness of the system in a stronger way.

\section{Preliminaries: equilibria and stability of a simple system with unilateral contact and Coulomb friction under constant loading}

\subsection{The simple model}

We consider the very classical mass-spring system in $\mathbb{I R}^{2}$ represented on Fig. 1 and studied in previous works, originally from the point of view of the existence and uniqueness of quasi-static solutions [9] and studied numerically in [1]. The particle of mass $m$ is connected to a rigid frame by two springs and constrained to move only above the horizontal axis.

Let $u_{t}$ and $u_{n}$ be respectively the tangential and the normal component of the displacement of the mass. When submitted to an external force of components $F_{t}$ and $F_{n}$, the trajectory of the mass is obtained by solving the following system where $\left({ }^{\circ}\right)$ indicates the derivative with respect to time:

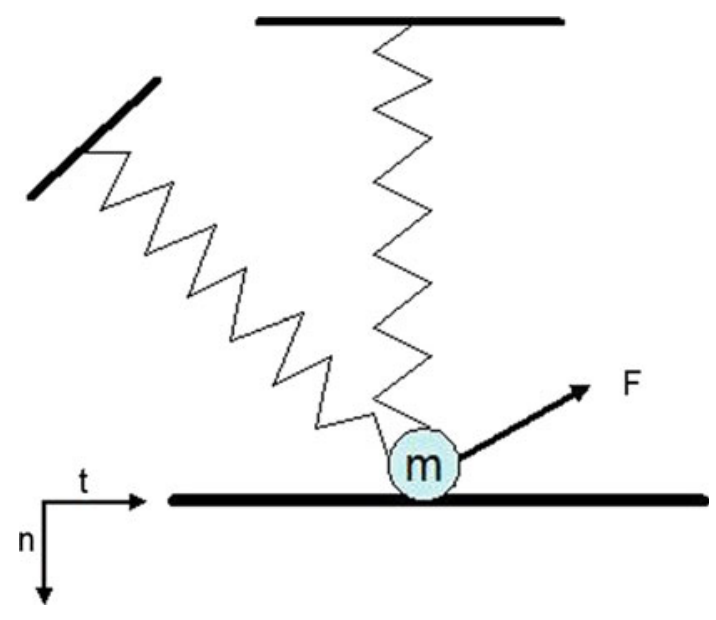

Fig. 1 The mass-spring system

$$
\left\{\begin{array}{l}
m \ddot{u}_{t}+K_{t} u_{t}+W u_{n}=F_{t}+R_{t}, \quad t>0 \\
m \ddot{u}_{n}+W u_{t}+K_{n} u_{n}=F_{n}+R_{n}, \\
u_{n} \leq 0, R_{n} \leq 0, u_{n} R_{n}=0, \\
\mu R_{n} \leq R_{t} \leq-\mu R_{n},\left\{\begin{array}{l}
\left|R_{t}\right|<\mu R_{n} \Longrightarrow \dot{u}_{t}=0, \\
\left|R_{t}\right|=\mu R_{n} \Longrightarrow \exists \lambda>0 \text { s.t. } \dot{u}_{t}=-\lambda R_{t} .
\end{array}\right.
\end{array}\right.
$$

to which one adds initial data compatible with the obstacle and an impact law with a restitution coefficient $e$ which reads: let $\tau$ be a contact time, then $\dot{u}_{n}\left(\tau^{+}\right)=-e \dot{u}_{n}\left(\tau^{-}\right)$. Such an impact law is well defined since $\dot{u}$ is a function of bounded variation. $K_{t}, K_{n}$ and $W$ are the stiffness coefficients of the system of linear springs represented on Fig. 1, $\mu$ is the friction coefficient, $R_{t}$ and $R_{n}$ are unknowns and denote respectively the tangential and normal components of the reaction of the obstacle. It has been shown, using tools given in [6] and [11], that this system is wellposed if the force is a piecewise analytical function of time [4]. As in previous papers (e.g. [12]), we stress the fact that any regularization of the friction law would not be in agreement with some fundamental physical requirements. In particular no motion must be initiated until the tangential reaction reaches a yield value and this yield value must depend on the normal reaction. The Coulomb friction law we have adopted seems to be the simplest friction law which satisfies these physical requirements.

\subsection{The set of equilibrium states}

It is useful for the present work to recall the set of equilibrium positions which has been fully determined in the case of a constant external force (see [5, 10]). The main qualitative results of this investigation are the following:

- the structure of the set of equilibria depends only on the sign of two quantities which are $K_{t} F_{n}-W F_{t}$ and 
$\mu-\frac{K_{t}}{W}$; the quantity $K_{t} F_{n}-W F_{t}$ which appears all through the paper shall be denoted by $\mathcal{A}$;

- equilibrium states without contact always exist when $\mathcal{A}<0$. The equilibrium state without contact is then unique. Moreover, if $\mu-\frac{K_{t}}{W}>0$, then the equilibrium state without contact coexists with infinitely many equilibrium states in contact with a strictly negative normal component of the reaction.

- If $\mathcal{A} \geq 0$, there are no equilibrium states without contact. Moreover the set of equilibria reduces to a single state in grazing contact if $\mathcal{A}=0$ and $\mu-\frac{K_{t}}{W}<0$.

- If $\mathcal{A}>0$ there always exist infinitely many equilibrium states, which all have a strictly negative normal component of the reaction. If in addition $\mu-\frac{K_{t}}{W} \geq 0$ then this set fills completely a half-line in the $\left\{R_{t}, R_{n}\right\}$ plane, while it fills only a bounded interval if $\mu-\frac{K_{t}}{W}<0$. In this case, the ends of the half-line or interval are in imminent sliding, while all the other equilibria are strictly stuck by friction.

\subsection{About stability}

As recalled in the introduction the first step of the qualitative analysis of the nonsmooth dynamical system we are studying delt with the classical notions of stability of equilibria. Using the fundamental result concerning the well-posedness of the Cauchy problem and having the complete description of the set of the equilibrium states, we chose initial data in the neighbourghoods of each given equilibrium state and then calculated whether the corresponding solution of problem (1) diverged or not from the equilibrium. This led to Lyapunov stability, asymptotic stability, or instability with classical definitions, and was performed for all the equilibria and all the values of the parameters [3].

More recently, we observed that the classical notions of stability are not suited to nonsmooth dynamics involving Coulomb friction. The basic observation leading to this is that when a contact point is strictly stuck by friction, that means has a reaction strictly inside the Coulomb cone, then the external force may vary without setting the point into motion as long as the reaction given by the equilibrium equation is not on the border of the cone. And the main theoretical result is that when a point is moving with a sliding velocity which reaches zero at some time, we are able to explicit sufficient conditions for the reaction to jump strictly inside the Coulomb cone and for the point to stay motionless for any future time. Of course the fact that we can change the forces without changing the position of the particle, or the fact that the particle can stop in finite time and remain at rest, can be seen as stability properties. This was stated in the form of a stability conjecture in a recent paper [13] and will be very useful in the present work.

\section{Existence of equilibrium states under oscillating loading}

We shall now study the mass-spring system of Fig. 1 up to the end of the paper. We assume that the parameters and the components of the external force are such that $\mu W<K_{t}$ and $\mathcal{A}=K_{t} F_{n}-W F_{t}>0$ i.e. the equilibria are all in strict contact (i.e. no grazing contact) and fill an interval in the $\left\{R_{t}, R_{n}\right\}$ plane, as represented in Fig. 2. All the equilibrium states are strictly stuck by friction except the two ends of the interval which are in imminent sliding, one to the right the other to the left. The purpose of the analysis consists in submitting the system to an additional oscillating force, and to study the effects of this perturbation on the equilibria.

If one of the strictly stuck equilibria represented in Fig. 2 is perturbed by a sufficiently small additional force, the mass remains at rest as long as the perturbation does not bring the reaction to the border of the cone, so that it is immediately seen that the normal component of the reaction of the equilibria belongs to an interval depending on time and given by:

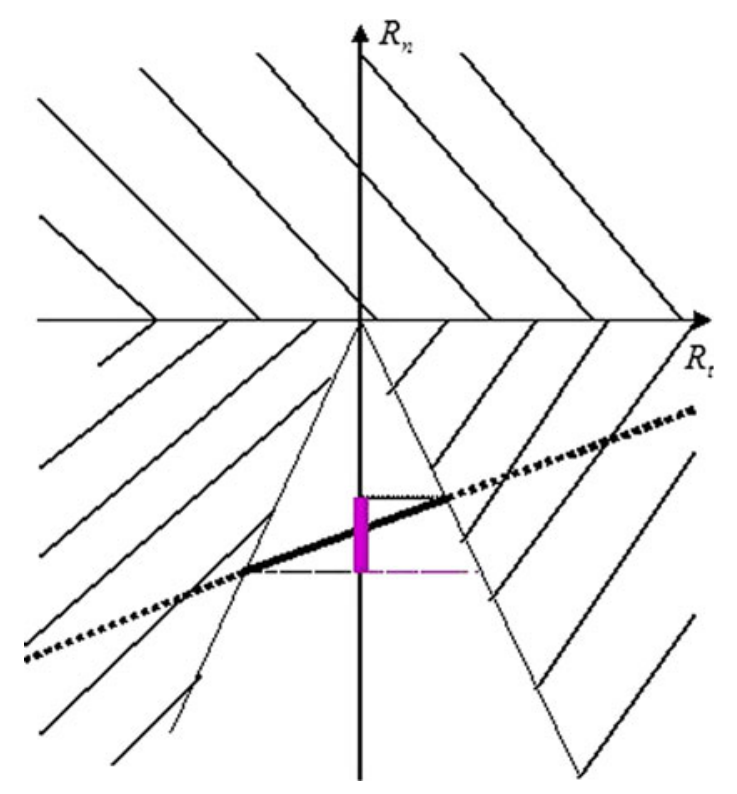

Fig. 2 The set of equilibrium states, for $\mathcal{A}>0$ and $\mu-\frac{K_{t}}{W}<0$. It is given by the intersection of the Coulomb cone with an affine manifold, here a straight line, which represents the equilibrium equation 
$\left\{R_{n}\right\}(t)=\left[\frac{-K_{t} F_{n}(t)+W F_{t}(t)}{K_{t}-\mu W}, \frac{-K_{t} F_{n}(t)+W F_{t}(t)}{K_{t}+\mu W}\right]$.

For any given $t$ the set $\left\{R_{n}\right\}(t)$ is the interval of the $R_{n}$ axis represented by a thick line in Fig. 2. The following result, the proof of which has been given in [13], will be a fundamental tool for a large part of the analysis:

Lemma 1 Let the loading be piecewise analytical and let $\left\{R_{n}\right\}(t)$ be the set of normal components of the reactions at time t corresponding to a strictly stuck equilibrium solution. We suppose that $\mathcal{A}>0$ and we consider the trajectory of a sliding mass which satisfies problem (1).

If at the instant $t^{*}$, when the mass stops sliding, its normal reaction $R_{n}^{*}$ belongs to the interior of $\left\{R_{n}\right\}\left(t^{*}\right)$, then the mass shall remain in a strictly stuck equilibrium state as long as its normal reaction belongs to the interior of $\left\{R_{n}\right\}(t)$.

Although problem (1) gives the dynamics in any situation, it will be useful to specify the equations of the trajectories during sliding periods. Inserting $u_{n} \equiv 0$ into Eq. (1) together with $R_{t}=\mu R_{n}$ yields the equation of the motion when sliding to the right:

$m \ddot{u}_{t}+\left(K_{t}-\mu W\right) u_{t}=F_{t}-\mu F_{n}$,

while $R_{t}=-\mu R_{n}$ yields the equation of the motion when sliding to the left:

$m \ddot{u}_{t}+\left(K_{t}+\mu W\right) u_{t}=F_{t}+\mu F_{n}$.

It is interesting to observe that the reactions, although varying according to strict Coulomb's friction law, have been eliminated from each equation so that each sliding phase is a part of a linear oscillation. The corresponding half-periods of the free oscillations, respectively denoted by $T_{\alpha}$ and $T_{\beta}$ for sliding to the right and to the left, are consequently given by:

$T_{\alpha}=\pi \sqrt{\frac{m}{K_{t}-\mu W}}, \quad T_{\beta}=\pi \sqrt{\frac{m}{K_{t}+\mu W}}$.

The frequencies $\omega_{\alpha}$ and $\omega_{\beta}$ shall also be introduced with obvious notations. The loadings $F_{t}(t)$ and $F_{n}(t)$ are now written in the following way:

$F_{t}(t)=F_{t}+P_{t}(t) \quad$ and $\quad F_{n}(t)=F_{n}+P_{n}(t)$,

where $F_{t}$ and $F_{n}$ are constant and $P_{t}(t)$ and $P_{n}(t)$ are tangential and normal perturbations.

We shall restrict our attention here to the case of a tangential perturbation, in other words to the case $P_{n}(t)=0$. It can be easily verified that, due to the coupling, the qualitative analysis is the same in the case of a perturbation with a non zero normal component. In fact the set of stationary solutions increases with the normal component of the perturbation.
In order to go on as far as possible with closed-form calculations, we are going to consider a tangential perturbation of rectangular wave shape given by, for $i=0,1$, $\ldots$ and $\varepsilon>0$ :

$P_{t}(t)=\varepsilon$ if $\left.\left.t \in\right] 2 i T,(2 i+1) T\right]$ and $P_{t}(t)=0$ if $\left.\left.t \in\right](2 i+1) T,(2 i+2) T\right]$.

So that $\varepsilon$ is the amplitude and $T$ the half period of the perturbation. On Fig. 3 the equilibrium solutions corresponding to $P_{t}(t)=0$ and to $P_{t}(t)=\varepsilon$ are represented in the $\left\{R_{t}, R_{n}\right\}$ plane. If we introduce, as in [13], the set:

$\bar{R}_{n}=\bigcap_{t>0}\left\{R_{n}\right\}(t)$,

we obtain in this case

$$
\begin{aligned}
\bar{R}_{n}= & {\left[\frac{-K_{t} F_{n}+W F_{t}}{K_{t}-\mu W}, \frac{-K_{t} F_{n}+W F_{t}}{K_{t}+\mu W}\right] } \\
& \cap\left[\frac{-K_{t} F_{n}+W\left(F_{t}+\varepsilon\right)}{K_{t}-\mu W}, \frac{-K_{t} F_{n}+W\left(F_{t}+\varepsilon\right)}{K_{t}+\mu W}\right], \\
= & {\left[\frac{-\mathcal{A}+\varepsilon W}{K_{t}-\mu W}, \frac{-\mathcal{A}}{K_{t}+\mu W}\right] . }
\end{aligned}
$$

On introducing the following notations that shall appear very often from now on: $R_{n}^{-}=\frac{-\mathcal{A}}{K_{t}-\mu W}, R_{n}^{+}=\frac{-\mathcal{A}}{K_{t}+\mu W}, R_{n \varepsilon}^{-}=$ $\frac{-\mathcal{A}+\varepsilon W}{K_{t}-\mu W}$ and $R_{n \varepsilon}^{+}=\frac{-\mathcal{A}+\varepsilon W}{K_{t}+\mu W}$ we have

$\bar{R}_{n}=\left[R_{n \varepsilon}^{-}, R_{n}^{+}\right]$.

This set $\bar{R}_{n}$ can be an interval on the $R_{n}$ axis (represented by a thick line in Fig. 3), can be reduced to a single point or can be empty depending on the value of $\varepsilon$. We shall discuss these different possibilities in the following. In order to illustrate our results, numerical calculations will also be

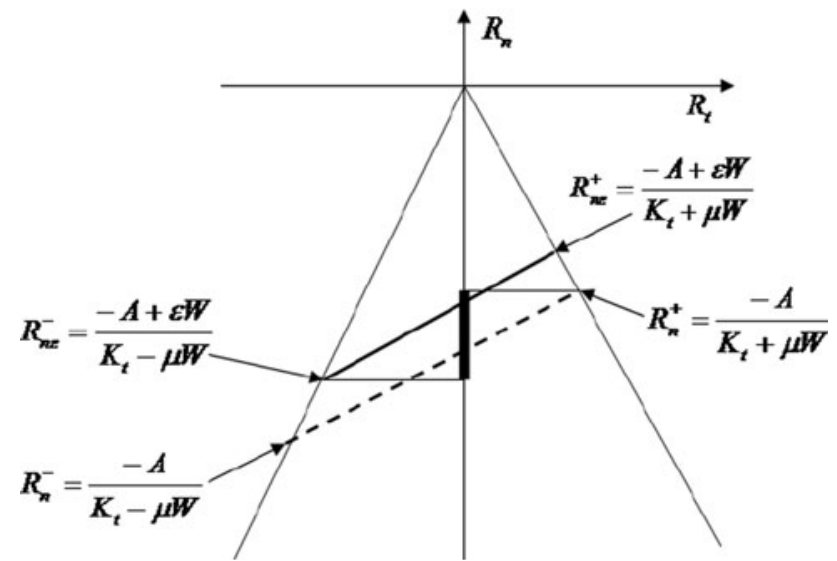

Fig. 3 Equilibrium solutions in the $\left\{R_{t}, R_{n}\right\}$ plane (dotted line equilibrium solutions when the tangential perturbation is equal to zero, full line equilibrium solutions when the tangential perturbation is equal to $\varepsilon$ ) 
performed with the software Maple, by using the following values for the data:

$m=1, \mu=0.5$, and $F_{n}=2, F_{t}=1, K_{t}=2$,

$W=1 \quad$ so that $\quad \mathcal{A}=3$.

From Eq. (2) we see that when $\varepsilon=\frac{2 \mu \mathcal{A}}{K_{t}+\mu W}$ the set $\bar{R}_{n}$ reduces to a single point, and the set of normal components of the reactions at equilibrium is a non zero measure interval when $\varepsilon<\frac{2 \mu \mathcal{A}}{K_{t}+\mu W}$ and an empty set when $\varepsilon>\frac{2 \mu \mathcal{A}}{K_{t}+\mu W}$. For example $\frac{2 \mu \mathcal{A}}{K_{t}+\mu W}=1.2$ when the above values of the data are used. We shall examine successively these different situations. As the parameter $m$ has no influence on this study, we choose from now on to take $m=1$.

\subsection{When $\varepsilon<\frac{2 \mu \mathcal{A}}{K_{t}+\mu W}$}

In this case the set $\bar{R}_{n}$ is a nonzero measure interval and we have the following result:

Proposition 1 When the amplitude $\varepsilon$ of the perturbing force is strictly smaller than $\frac{2 \mu \mathcal{A}}{K_{t}+\mu W}$ :

1. there exists infinitely many equilibrium solutions,

2. all sliding trajectories attain an equilibrium in finite time.

Proof The first point is a direct consequence of Lemma (1), indeed any position in the interval $\left[\frac{R_{n e}^{-}+F_{n}}{W}, \frac{R_{n}^{+}+F_{n}}{W}\right]$ with a zero velocity satisfies problem (1).

The proof of point 2 is divided into several steps. We shall just give the steps and drop the calculations which are not difficult. At first we show that when the initial data are sufficiently close to equilibrium and $T$ is sufficiently large, an equilibrium is reached after only one sliding oscillation. Indeed, let us look at the solution of the following system which describes a first phase of sliding to the right until the velocity is zero, a second phase during which the particle stays at rest until the end of the half period, a third phase of sliding to the left and a last phase of rest until the end of the period.

$$
\left\{\begin{array}{l}
T>T_{\alpha}, \frac{2 R_{n \varepsilon}^{-}-R_{n \varepsilon}^{+}+F_{n}}{W} \leq u_{0}<\frac{R_{n \varepsilon}^{-}+F_{n}}{W}, \\
\ddot{u}_{1}+\omega_{\alpha}^{2} u_{1}=F_{t}-\mu F_{n}+\varepsilon, t \in\left(0, T_{\alpha}\right) \\
u_{1}(0)=u_{0}, \dot{u}_{1}(0)=0, \\
\dot{u}_{2}(t)=0, t \in\left(T_{\alpha}, T\right) \\
\ddot{u}_{3}+\omega_{\beta}^{2} u_{3}=F_{t}+\mu F_{n}, t \in\left(T, T+T_{\beta}\right) \\
u_{1}(T)=u_{3}(T), \dot{u}_{3}(T)=0, \\
\dot{u}_{4}(t)=0, t \in\left(T+T_{\beta}, 2 T\right) .
\end{array}\right.
$$

If $u_{0} \in\left[\frac{2 R_{n \varepsilon}^{-}-R_{n}^{+}+F_{n}}{W}, \frac{R_{n}^{-}+F_{n}}{W}\left[\right.\right.$ then $u_{1}(T) \in\left[\frac{R_{n \varepsilon}^{-}+F_{n}}{W}, \frac{R_{n}^{+}+F_{n}}{W}\right]$ so that $u_{2} \equiv u_{3} \equiv u_{4}$ and the result is triviat.

If on the other hand $u_{0} \in\left[\frac{2 R_{n e}^{-}-R_{n e}^{+}+F_{n}}{W}, \frac{2 R_{n e}^{-}-R_{n}^{+}+F_{n}}{W}\right.$ [ then inserting the solution of (8) into the expression of the normal component of the reaction given by the equilibrium equations gives

$$
\begin{aligned}
R_{n}(2 T)-R_{n}(0) & =2 \frac{2 \mu W \mathcal{A}-\varepsilon W\left(K_{t}+\mu W\right)}{K_{t}^{2}-\mu^{2} W^{2}} \\
& =2\left(R_{n}^{+}-R_{n \varepsilon}^{-}\right),
\end{aligned}
$$

which means that $R_{n}(2 T) \in \bar{R}_{n}$.

Let us now look at the case where $u_{0} \notin$ $\left[\frac{2 R_{n e}^{-}-R_{n e}^{+}+F_{n}}{W}, \frac{R_{n e}^{-}+F_{n}}{W}[\right.$. We shall show that the trajectory will enter in finite time into the interval $\left[\frac{2 R_{n \varepsilon}^{-}-R_{n \varepsilon}^{+}+F_{n}}{W}, \frac{R_{n \varepsilon}^{-}+F_{n}}{W}\right]$ with a zero velocity so that we come back to the previous case. This is a consequence of the following result:

Lemma 2 Assume $T>T_{\alpha}$ and a trajectory is such that there exists some $\bar{t}$ such that

$\left\{\begin{array}{l}\dot{u}(\vec{t})=0 \\ \dot{u}(t) \leq 0 \text { for } t \in[\bar{t}-\eta, \bar{t}], \dot{u}(t) \geq 0 \text { for } t \in[\bar{t}, \bar{t}+\eta],\end{array}\right.$

Then, $\exists \hat{t}, \bar{t}<\hat{t}<\bar{t}+2 T$, such that:

$\left\{\begin{array}{l}\dot{u}(\hat{t})=0 \\ \dot{u}(t) \leq 0 \text { for } t \in[\hat{t}-\eta, \hat{t}], \dot{u}(t) \geq 0 \text { for } t \in[\hat{t}, \hat{t}+\eta],\end{array}\right.$

The proof of Lemma 2 is elementary for $\bar{t}=0$, and is adapted with simple estimates for larger $\bar{t}$.

We then get that

$2\left(R_{n}^{+}-R_{n \varepsilon}^{-}\right) \leq R_{n}(\hat{t})-R_{n}(\bar{t}) \leq R_{n \varepsilon}^{-}-R_{n}(\bar{t})$,

which gives the result.

The case where $T<T_{\alpha}$ must then be studied. We have already proved in [13] that starting in imminent sliding and if the amplitude $\varepsilon$ is small enough $\left(\varepsilon \leq \frac{\mu \mathcal{A}}{K_{t}+\mu W}\right)$, the trajectory leads to an equilibrium state after sliding in only one direction (i.e. the velocity keeps the same sign up to the final stop) as is the case on Fig. 4a. The proof of the same result is easily adapted if the trajectory starts from an initial data out of equilibrium. If $\varepsilon$ is larger than $\frac{\mu \mathcal{A}}{K_{t}+\mu W}$, then Lemma 2 is easily adapted and again an inequality similar to (9) gives the result.

Using the values introduced in (7) and taking $\varepsilon=$ $1<\frac{2 \mu \mathcal{A}}{K_{t}+\mu W}=1.2$, examples of trajectories are represented in the phase space $\left\{u_{t}, \dot{u}_{t}\right\}$ in Figs. 4 and 5.

3.2 When $\varepsilon=\frac{2 \mu \mathcal{A}}{K_{t}+\mu W}$

This value of $\varepsilon$ is the transition between the existence of equilibrium states without non trivial periodic solutions 


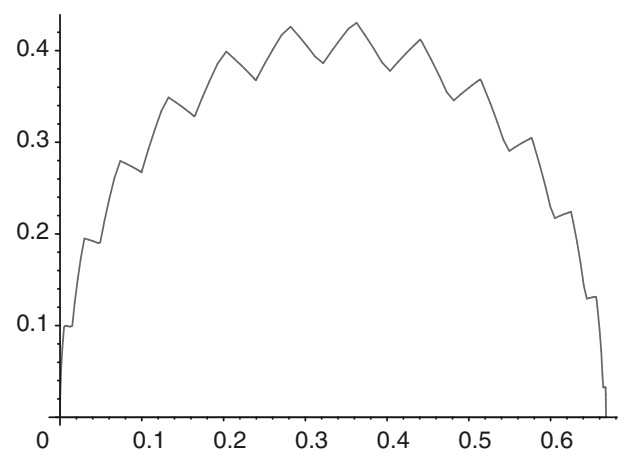

(a) $\varepsilon=1$ and $T=0.1$

Fig. 4 Evolution in the phase space $\left(u_{t}, \dot{u}_{t}\right)$ when $\varepsilon=1$ and $T$ is small

and periodic solutions without equilibria. The interval $\bar{R}_{n}$ is reduced to a single point which means, due to Lemma 1 that there exists a unique equilibrium state whatever the value of the period of the excitation. Using the numerical values given in (7), this equilibrium state is the point $\left(u_{t}=0.8, \dot{u}_{t}=0\right)$.

A constructive proof, which is elementary in this case but which is at the origin of proofs for solutions in less trivial cases, shows the existence of periodic solutions. Assume the particle is sliding to the right and the half

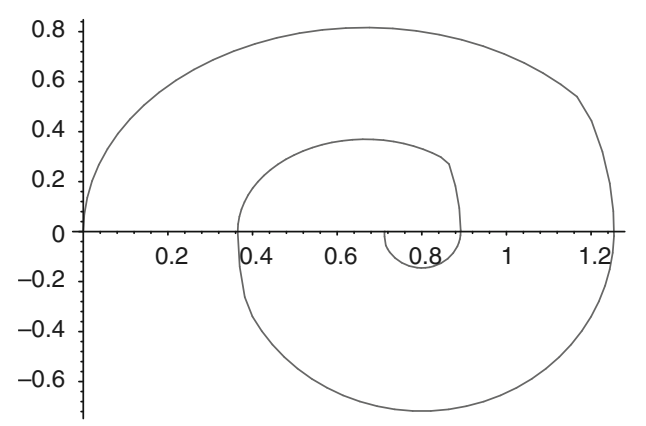

(a) $\varepsilon=1$ and $T=2$

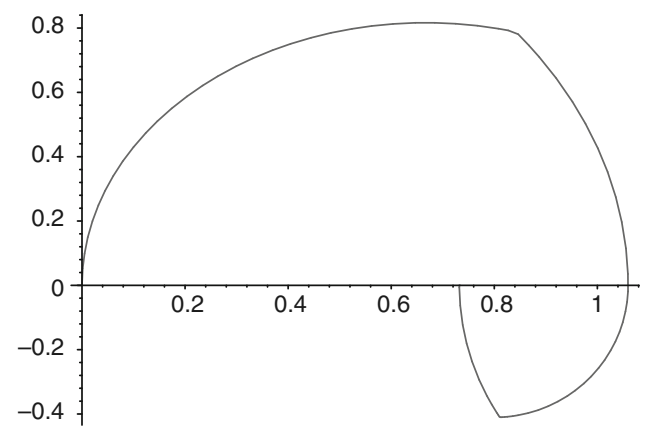

(b) $\varepsilon=1$ and $T=1.5$

period $T$ of the excitation is larger than the half sliding period $T_{\alpha}$. Then the sliding motion will stop before the perturbation of amplitude $\varepsilon$ is removed, so that, according to lemma 1 the particle shall remain in a strictly stuck equilibrium if the normal component of the reaction belongs to the set given by Eq. (2) at that time (equal to $\left[R_{n \varepsilon}^{+}, R_{n \varepsilon}^{-}\right]$in this case). Then the amplitude of the perturbation jumps from $\varepsilon$ to zero, so that the particle is no longer at equilibrium and slides to the left. Since $T_{\alpha}>T_{\beta}$, the half period $T$ of the excitation is also larger than the half sliding

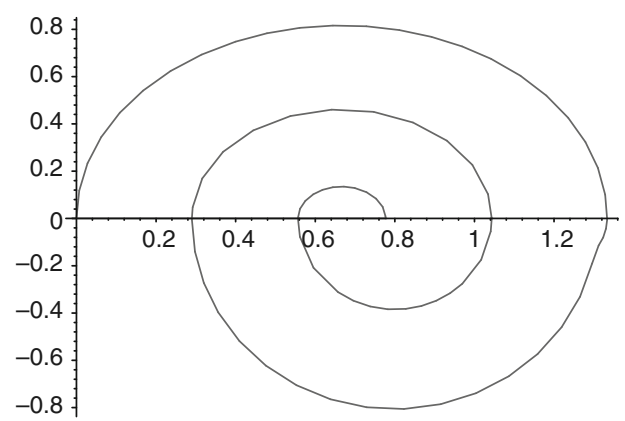

(b) $\varepsilon=1$ and $T=3$

Fig. 5 Evolution in the phase space $\left(u_{t}, \dot{u}_{t}\right)$ when $\varepsilon=1$

Fig. 6 A periodic solution when $\varepsilon=\frac{2 \mu \mathcal{A}}{K_{t}+\mu W}$

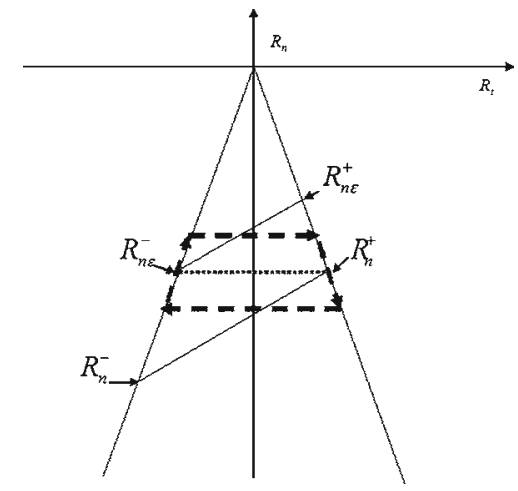

(a) In the $\left\{R_{t}, R_{n}\right\}$ plane

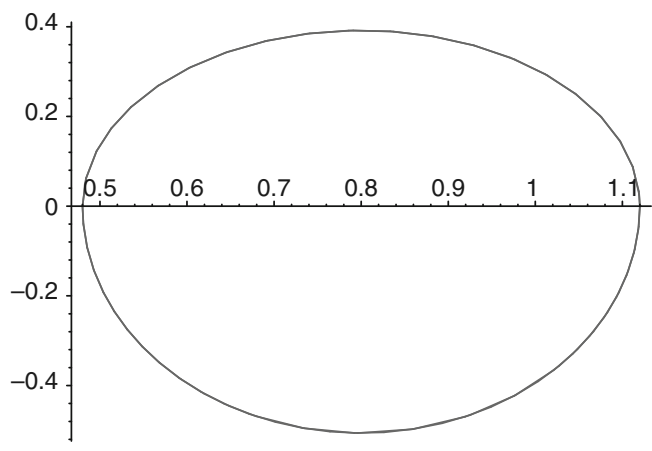

(b) In the phase space 
period $T_{\beta}$. Again the particle stops and remains at rest with a reaction inside the cone up to the moment when the perturbation jumps to $\varepsilon$ again. This will give a periodic trajectory if the position at the second stop is equal to the position at the initial data. Such trajectories are represented in the $\left\{R_{t}, R_{n}\right\}$ plane on Fig. 6a, and in the phase space on Fig. $6 \mathrm{~b}$ where we can see that they are diffeomorphic to ellipses.

According to Eqs. $(3,4)$ and to the assumptions on the loading, solutions of this type do exist if the following problem has a solution:

$$
\left\{\begin{array}{l}
\ddot{u}_{1}+\left(K_{t}-\mu W\right) u_{1}=F_{t}+\varepsilon-\mu F_{n}, \\
u_{1}(0)=u_{0}, \dot{u}_{1}(0)=0, \\
\tilde{u}_{1}=u_{1}(\tilde{t}), \tilde{t} \text { such that } \tilde{t} \neq 0, \dot{u}_{1}(\tilde{t})=0 \\
\ddot{u}_{2}+\left(K_{t}+\mu W\right) u_{2}=F_{t}+\mu F_{n}, \\
u_{2}(T)=\tilde{u}_{1}, \dot{u}_{2}(T)=0 \\
\hat{u}_{2}=u_{2}(\hat{t}), \hat{t} \text { such that } \hat{t} \neq 0, \dot{u}_{2}(\hat{t})=0 \\
\hat{u}_{2}=u_{0}
\end{array}\right.
$$

After some elementary calculations, we get that this system has a solution if and only if $\varepsilon=\frac{2 \mu \mathcal{A}}{K_{t}+\mu W}$ and $u_{0} \in$ $\left[\frac{F_{n}+2 R_{n}^{\varepsilon-}-R_{n}^{\varepsilon+}}{W}, \frac{F_{n}+R_{n}^{\varepsilon-}}{W}\right]$ (for the data chosen in (7) we have $\left.u_{0} \in[0.32,0.8]\right)$. Obviously this construction works for any $T$ larger that $T_{\alpha}$. Represented in the phase space all these trajectories have the same center at the equilibrium point $\left(u_{t}=\frac{F_{n}+R_{n}^{\varepsilon-}}{W}, \dot{u}_{t}=0\right)$. Moreover, numerical experiments show that trajectories starting from any initial data outside the outer periodic solution always reach a periodic solution. These observations suggest a stability property of the set of periodic solutions, and clearly a Lyapunov stability of the equilibrium.

We have thus proved the following qualitative features:

\section{Proposition 2}

- When the amplitude $\varepsilon$ of the perturbing force is equal to $\frac{2 \mu \mathcal{A}}{K_{t}+\mu W}$ and the half period $T$ is smaller than $T_{\alpha}$, all the trajectories go to the single equilibrium of the system.

- When the amplitude $\varepsilon$ of the perturbing force is equal to $\frac{2 \mu \mathcal{A}}{K_{t}+\mu W}$ and the half period $T$ is larger than $T_{\alpha}$, infinitely many periodic solutions of period $2 T$ appear around the equilibrium which behaves locally as a center.

Remark 1 Concerning Proposition 2 it is interesting to note that:

- The equilibrium state is reached at infinity, while one equilibrium among a whole set of equilibria was always reached in finite time for smaller values of $\varepsilon$.

- The amplitude of the oscillations takes more and more time to decrease as $T$ increases towards $T_{\alpha}$, and beyond $T_{\alpha}$ these oscillations change into periodic solutions. This suggests that the point $(T, \varepsilon)=\left(T_{\alpha}, \frac{2 \mu \mathcal{A}}{K_{t}+\mu W}\right)$ could be interpreted as a Hopf bifurcation point.

- Such a Hopf type bifurcation point is nevertheless not classical since all the periodic solutions lie in a bounded domain diffeomorphic to an ellipse and are surrounded by non periodic solutions similar to those obtained before the bifurcation point but converging here towards a periodic solution. This is represented on Fig. 7.

\section{When no equilibrium solutions exist}

All the remaining part of this work deals with the range $\varepsilon>\frac{2 \mu \mathcal{A}}{K_{t}+\mu W}$. This range will be divided into two parts separated by a relatively complicated boundary: a «lower» range where the particle is always in contact, and an «upper» range, where at least one point of the trajectory looses contact. In the present work we shall carry out two steps of the analysis of the qualitative dynamics: in the range where the trajectories are always in contact, we shall focus on the existence of periodic solutions giving a detailled description of their different shapes. Then special attention will be paid to the upper boundary of this range defined as the value of $\varepsilon$ corresponding to the occurence of the loss of contact. This boundary will be shown to depend strongly on the period of the excitation. Trajectories which

Fig. 7 Solutions for $\varepsilon=1.2$

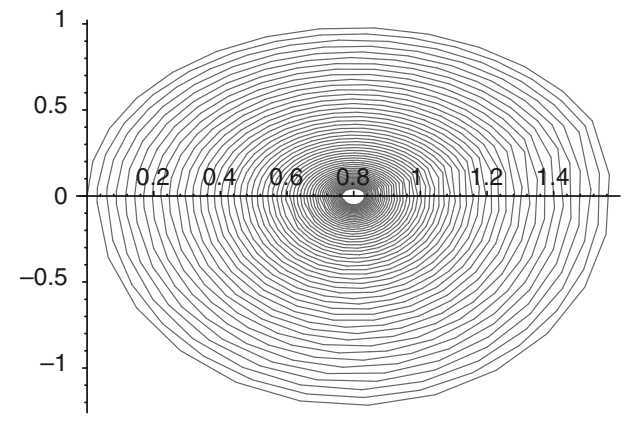

(a) Convergence towards the single stationnary solution for $T<T_{\alpha}$

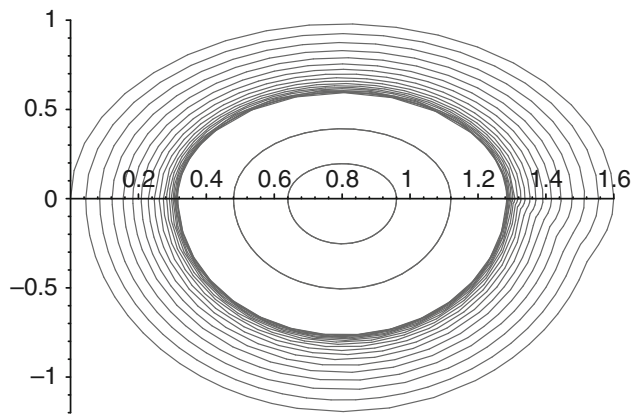

(b) Periodic and non periodic solutions for $T>T_{\alpha}$ 
loose contact, therefore involve impacts, shall be studied in a future work. The qualitative behaviour of the dynamics is conveniently described, in the period-amplitude plane, by the diagram depicted in Fig. 10a.

We first prove that periodic solutions of different kinds and multiplicity exist for any $T$ as soon as there no longer exist equilibrium states. Due to the nonsmoothness of system (1), the method we use for proving the existence of periodic solution will consist in following the solution by piecewise calculations for given values of the parameters and proving that there exist solutions for which the trajectory comes back to its initial data. Of course this ensures the existence of some periodic solutions, but does not prove that there does not exist others. In some cases nevertheless the well-posedness of the Cauchy problem shall enable us to exclude other periodic solutions. The mechanical parameters will be fixed in the whole analysis, with the explicit values given in (7) for numerical computations, and we shall explore the $\{T, \varepsilon\}$ plane. As a last point, let us mention that in all this section numerical experiments were used as a guide for the theoretical calculations we subsequently performed (see e.g. [7]).

The analysis will be broken into several ranges of the half period $T$ (see Fig. 10a), $T>T_{\alpha}+T_{\beta}$ where we shall in particular find inifinitely many periodic solutions of period $4 T, T_{\beta}<T<T_{\alpha}+T_{\beta}$ which will appear as relatively intricate, and $T<T_{\beta}$.

\subsection{Periodic solutions for large $T$.}

The fact that the data $T$ is sufficiently large allows a generalization of the method used in Sect. 3.2. This leads to calculations based upon the following remarks:

\section{Remark 2}

- Since the period is sufficiently large, the explicit solution in each part of the trajectory is the same as if the loading were a constant.

- In each given sliding phase with initial data $\left\{u_{0}, v_{0}\right\}$ with $u_{0}$ out of equilibrium and $v_{0}=0$, the sliding motion has a first stop at a point $u_{1}$ symmetrical of $u_{0}$ with respect to the equilibrium point in imminent sliding in the same direction, which is nothing but the elementary property of a linear oscillator with constant right hand side and constant coefficients.

- Moreover, one can indifferently use $u_{t}$ or $R_{n}$ as the basic unknown of the analysis, since for a sliding motion, the position $u_{t}$ is connected to the normal component of the reaction $R_{n}$ by the equilibrium equation which reduces here to: $W u_{t}=F_{n}+R_{n}$, and, as the period of the excitation is sufficiently large, the particle can always stop before being set into motion again.
We are going to build a periodic solution in the $\left\{R_{t}, R_{n}\right\}$ plane. Let $R_{n}^{\varepsilon-}$ be the normal component of the reaction of the equilibrium state in imminent sliding to the right when $P_{t}(t)=\varepsilon$, and we take $R_{n}^{\varepsilon-}-x$ (with $x>0$ ) as initial data with zero velocity. $T$ larger than $T_{\alpha}$ implies that the velocity goes through zero at the point $R_{n}^{\varepsilon-}+x$. Assume in addition that $x$ is such that $R_{n}^{\varepsilon-}+x$ is larger than $R_{n}^{\varepsilon+}$, where $R_{n}^{\varepsilon+}$ is the reaction of the equilibrium state in imminent sliding to the left still with $P_{t}(t)=\varepsilon$, and let $R_{n}^{\varepsilon-}+x=R_{n}^{\varepsilon+}+y, y>0$. Then the reaction jumps to the other side of the cone and the particle slides to the left during a half-period $T_{\beta}$ up to a stop since $T>T_{\alpha}+T_{\beta}$. The reaction then jumps to an equilibrium state, with $R_{n}=R_{n}^{\varepsilon+}-y$ and stays there until the change of the external load at time $T$, when $P_{t}(t)$ is set to zero. Let then $R_{n}^{\varepsilon+}-y=R_{n}^{+}+z, z>0$. At time $T$ the particle is set into motion again and slides to the left during a period $T_{\beta}$ up to a stop at the reaction $R_{n}^{+}-z$ and then jumps to an equilibrium and stays there until the time $2 T$ where the data will be set to $P_{t}(t)=\varepsilon$ again. Obviously, such a trajectory is periodic with period $2 T$ if the condition $R_{n}^{+}-z=R_{n}^{\varepsilon-}-x$ is satisfied.

Substituting $z$ and $y$ in this periodicity condition gives an equation for the initial data:

$$
\begin{aligned}
R_{n}^{\varepsilon-}-x & =R_{n}^{+}-z \\
& =2 R_{n}^{+}-R_{n}^{\varepsilon+}+y \\
& =2 R_{n}^{+}-2 R_{n}^{\varepsilon+}+R_{n}^{\varepsilon-}+x,
\end{aligned}
$$

so that

$x=R_{n}^{\varepsilon+}-R_{n}^{+}=\frac{\varepsilon W}{K_{t}+\mu W}$.

This trajectory exists if it is compatible with the description we have given above, in other words if $0 \leq y \leq R_{n}^{\varepsilon+}-R_{n}^{\varepsilon-}$. So that finally we have the existence of this unique periodic solution only when $\frac{2 \mu \mathcal{A}}{K_{t}+\mu W} \leq \varepsilon \leq \frac{4 \mu \mathcal{A}}{K_{t}+3 \mu W}$. This trajectory is represented on Fig. $8 \mathrm{a}$ in the $\left\{R_{t}, R_{n}\right\}$ plane, and on Fig. $8 \mathrm{~b}$ in the phase space, where we observe that because of the successive stops, the trajectory is no longer diffeomorphic to an ellipse. For a given value of the pair $\left(u_{0}, v_{0}\right)$, due to the well-posedness of the Cauchy problem there does not exist other trajectories passing through this initial data.

In a similar way, if we remove the periodicity condition $R_{n}^{+}-z=R_{n}^{\varepsilon-}-x$ we can obtain a periodic solution of period $4 T$ by doing two complete loops instead of one. using the same definition as above for $x, y$ and $z$ and introducing the quantities: $w=R_{n}^{\varepsilon-}-\left(R_{n}^{+}-z\right), q=R_{n}^{\varepsilon-}+w-R_{n}^{\varepsilon+}$ and $u=R_{n}^{\varepsilon+}-q-R_{n}^{+}$for the second loop, a periodic solution of period $4 T$ exists if and only if $R_{n}^{+}-u=R_{n}^{\varepsilon-}-x$. A simple computation shows that the periodicity condition is true for any compatible value of $x$, that is for any value of $x$ implying values of $y, z$ and $q$ compatible with the above construction. 
Fig. 8 Trajectories of solutions of period $2 T$ for $\varepsilon=1.5$ and $T=4.6$ (larger than $\left.T_{\alpha}+T_{\beta}\right)$

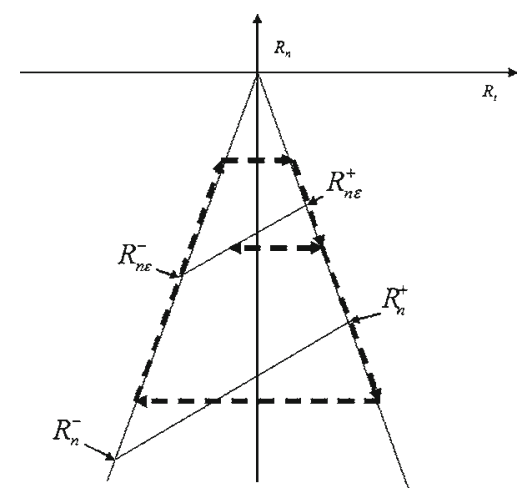

(a) In the $\left(R_{t}, R_{n}\right)$ plane

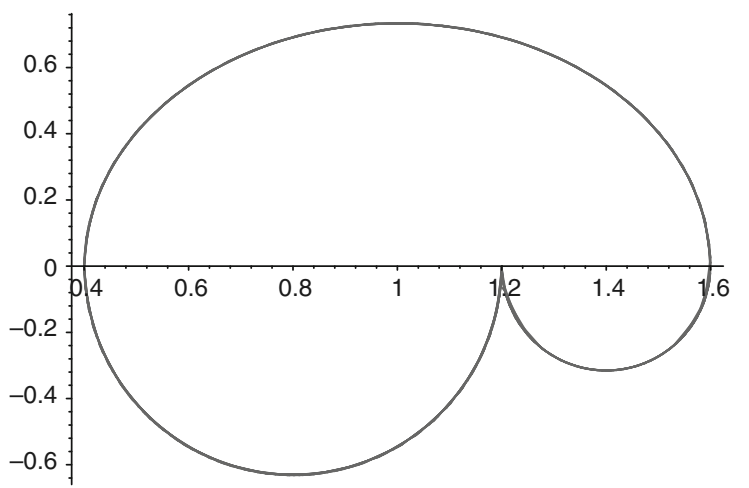

(b) In the phase space

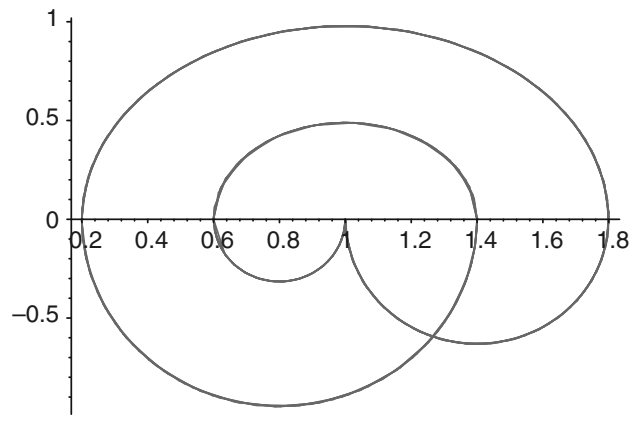

(a) $u_{t 0}=0.2\left(\right.$ or $\left.u_{t 0}=0.6\right)$

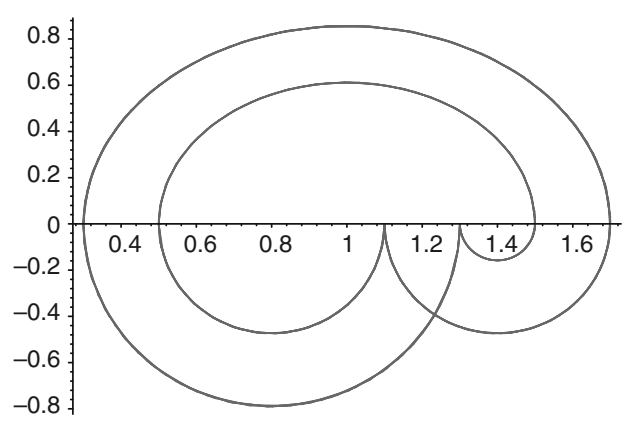

(b) $u_{t 0}=0.3\left(\right.$ or $\left.u_{t 0}=0.5\right)$

Fig. 9 Trajectories in the phase space $\left(u_{t}, \dot{u}_{t}\right)$ of solutions of period $4 T$ for $\varepsilon=1.5$ and $T=4.6$ (larger than $T_{\alpha}+T_{\beta}$ )

So that this time not only must we have $0 \leq y \leq R_{n}^{\varepsilon+}-$ $R_{n}^{\varepsilon-}$ but also $0 \leq z \leq R_{n}^{+}-R_{n}^{-}$and $y \leq q \leq R_{n}^{\varepsilon+}-R_{n}^{\varepsilon-}$. By a straightforward but rather tedious computation, these three conditions are shown to imply that all values of $x$ belonging to a given interval correspond to a periodic solution of period $4 T$. If $K_{t} \leq 5 \mu W$ this interval is given by:

$\max \left(R_{n}^{\varepsilon+}+R_{n}^{\varepsilon-}, 2\left(R_{n}^{\varepsilon-}+R_{n}^{+}\right) \leq x \leq R_{n}^{\varepsilon+}+R_{n}^{+}\right.$.

The case $K_{t}>5 \mu \mathrm{W}$ would lead to a smaller interval obtained by a similar calculation.

In both cases this interval of possible values for $x$ is not empty as long as $\frac{2 \mu \mathcal{A}}{K_{t}+\mu W} \leq \varepsilon \leq \frac{4 \mu \mathcal{A}}{K_{t}+3 \mu W}$.

We have thus established the following result:

Proposition 3 For all $T \geq T_{\alpha}+T_{\beta}$,

- if $\varepsilon \in\left[\frac{2 \mu \mathcal{A}}{K_{t}+\mu W}, \frac{4 \mu \mathcal{A}}{K_{t}+3 \mu W}\right]$, there exists a unique periodic solution of period $2 T$ obtained with the initial data $u_{0}=\left(F_{n}+R_{n}^{\varepsilon-}-R_{n}^{\varepsilon+}+R_{n}^{+}\right) / W$ and $v_{0}=0$,

- if $\varepsilon \in] \frac{2 \mu \mathcal{A}}{K_{t}+\mu W}, \frac{4 \mu \mathcal{A}}{K_{t}+3 \mu W}[$, there exists infinitely many periodic solutions of period $4 T$ obtained with any inital data $u_{0}$ in a bounded nonzero measure interval and $v_{0}=0$.

\section{Remark 3}

- The condition $K_{t}>\mu W$ ensures that the interval ]$\frac{2 \mu \mathcal{A}}{K_{t}+\mu W}, \frac{4 \mu \mathcal{A}}{K_{t}+3 \mu W}[$ is not empty.

- If for example $K_{t} \leq 5 \mu W$, the nonzero measure interval of Proposition 3 is $]\left(F_{n}+R_{n}^{\varepsilon-}-R_{n}^{\varepsilon+}+\right.$ $\left.R_{n}^{+}\right) / W,\left(F_{n}+R_{n}^{\varepsilon-}-\max \left(R_{n}^{\varepsilon+}+R_{n}^{\varepsilon-}, 2\left(R_{n}^{\varepsilon-}+R_{n}^{+}\right)\right) /\right.$ $W]$.

- The lower bound of this interval corresponds to the unique periodic solution of period $2 T$.

- If for any pair $(\varepsilon, T)$ in the range studied in Proposition 3 , we choose an initial data which does not belong to the nonzero measure interval we observe numerically that the solution is not periodic but converges to one of the periodic solutions of period $4 T$ in finite time.

Exemples of trajectories of period $4 T$ are represented on Fig. 9.

\subsection{Investigation for decreasing values of $T$}

When $T$ becomes smaller than $T_{\alpha}+T_{\beta}$, the qualitative behaviour of the oscillator becomes more intricate than for larger values of $T$. This qualitative behaviour that we shall establish in the following is summarized on Fig. 10b which 
represents the central part of the $\{T, \varepsilon\}$ plane (see Fig. 10a). Six zones appear that we shall first describe qualitatively.

- $\boldsymbol{\Omega}_{1}$ : The periodic solutions represented on Figs. 8 and 9 no longer exist when $T<T_{\alpha}+T_{\beta}$, but we shall nevertheless prove the existence of periodic solutions of period $2 T$ when $\mathrm{T}$ is close to $T_{\alpha}+T_{\beta}$ by a direct analytical calculation.

- $\boldsymbol{\Omega}_{2}$ : A qualitative change appears on the left of $\Omega_{1}$ by the occurence of a phase difference between the loading and the periodic solutions.

- $\boldsymbol{\Omega}_{3}$ : The left boundary of $\Omega_{2}$ corresponds to the loss of existence of sliding solutions, so that $\Omega_{3}$ contains solutions which loose contact. They exist even for small values of $\varepsilon$.

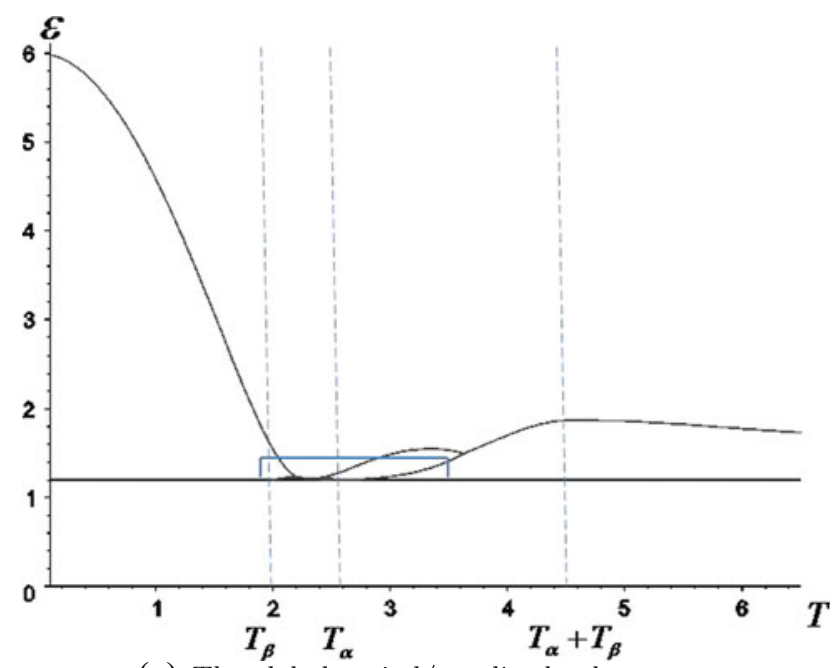

(a) The global period/amplitude plane

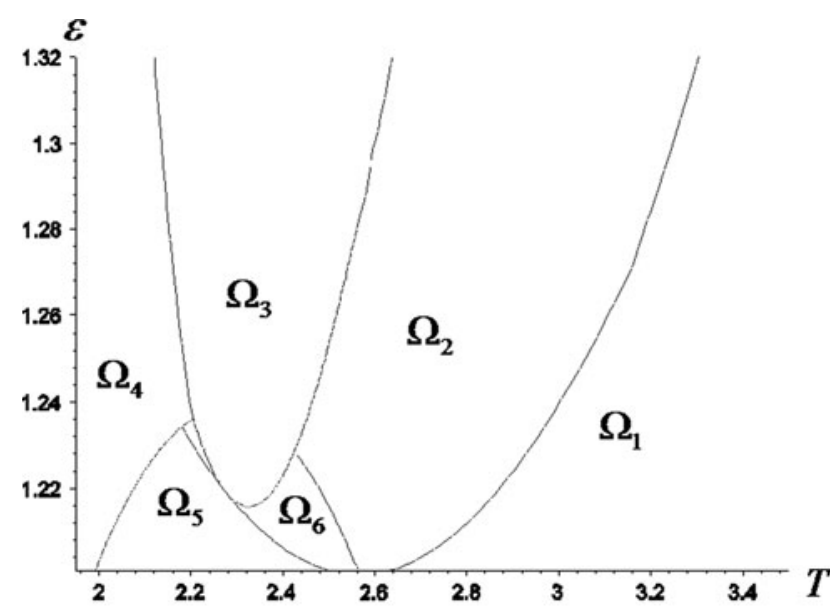

(b) A zoom, with an important vertical expansion, of the central part inside the rectangle

Fig. 10 Qualitative behaviour in the central part of the $\{T, \varepsilon\}$ plane
- $\boldsymbol{\Omega}_{\mathbf{4}}$ : This region is bounded on the left by the axis $T=0$ and contains periodic solutions which are still out of phase with respect to the loading.

- $\boldsymbol{\Omega}_{5}$ and $\boldsymbol{\Omega}_{\mathbf{6}}$ : Between $\boldsymbol{\Omega}_{2}$ and $\boldsymbol{\Omega}_{4}$, are found very small zones referred to as $\Omega_{5}$ and $\Omega_{6}$ which contain other types of periodic solutions. Locally the structure of the $\{T, \varepsilon\}$ plane is relatively intricate but going through $\Omega_{5}$ and $\Omega_{6}$, the transition from $\Omega_{2}$ to $\Omega_{4}$ appears as a continuous deformation of the orbits.

With obvious notations we call $\Gamma_{i j}$ the common boundary between $\Omega_{\mathrm{i}}$ and $\Omega_{\mathrm{j}}$.

\subsubsection{Zone $\Omega_{1}:$ T close to $T_{\alpha}+T_{\beta}$}

In this range of half periods of the excitation smaller than those studied in the previous subsection, the case of $T$ sufficiently close to $T_{\alpha}+T_{\beta}$ will be of special interest since, although the previous simple analysis no longer applies, we still obtain the existence of a single periodic solution with an explicit formula for the corresponding initial data. This enables us to obtain explicitely the limit of the range in the $\{T, \varepsilon\}$ plane, where these solutions exist. Moreover, we shall see how new qualitative changes appear in the trajectory as $T$ decreases. According to Remark 2, we now use the sliding position as the unknown of the dynamical problem, instead of $R_{n}$. Let $u^{\varepsilon-}$ and $u^{-}$be the positions of the equilibrium state in imminent sliding to the right respectively for $P(t)=\varepsilon$ and for $P(t)=0$, and let the initial data be $\left\{u_{0}, v_{0}\right\}:=\left\{u^{\varepsilon-}-x, 0\right\}$ for some unknown positive $x$. Since $T>T_{\alpha}$, the first part of the trajectory leads to a first stop at $u_{1}=u^{\varepsilon-}+x$ for $t=T_{\alpha}$ where there is a jump of the reaction to the other side of the cone. From this time, the remaining part of the trajectory is the solution to the following system:

$$
\left\{\begin{array}{l}
\ddot{u}_{2}+\omega_{\beta}^{2} u_{2}=F_{t}+\mu F_{n}+\varepsilon, t \in\left(T_{\alpha}, T\right) \\
u_{2}\left(T_{\alpha}\right)=u_{1}, \dot{u}_{2}\left(T_{\alpha}\right)=0, \\
\ddot{u}_{3}+\omega_{\beta}^{2} u_{3}=F_{t}+\mu F_{n}, t \in(T, \tilde{t}) \\
u_{3}(T)=u_{2}(T), \dot{u}_{3}(T)=\dot{u}_{2}(T), \\
\tilde{u}=u_{3}(\tilde{t}), \tilde{t} \text { such that } \dot{u}_{3}(\tilde{t})=0 .
\end{array}\right.
$$

We add the periodicity condition given by $\tilde{u}=u_{0}$. Going through the calculations we obtain one and only one value of $x$ given by the explicit formula:

$x=\frac{\frac{\varepsilon}{\omega_{\beta}^{2}}\left[u^{\varepsilon-}-u^{\varepsilon+}\right]\left(1-\cos \omega_{\beta}\left(T-T_{\alpha}\right)\right)}{2\left[u^{\varepsilon-}-u^{\varepsilon+}\right]+\frac{\varepsilon}{\omega_{\beta}^{2}}\left(1+\cos \omega_{\beta}\left(T-T_{\alpha}\right)\right)}$,

where $u^{\varepsilon-}=\frac{F_{t}-\mu F_{n}+\varepsilon}{\omega_{2}^{2}}$ and $u^{\varepsilon+}=\frac{F_{t}+\mu F_{n}+\varepsilon}{\omega_{\beta}^{2}}$.

Due to the well-posedness of the Cauchy problem, no other trajectory passes through the point $\left(u_{0}, 0\right)$. 
From formula (15) we get the periodic solution of period $2 T$ for any $\varepsilon$ in the corresponding range and $T$ smaller than, but close to, $T_{\alpha}+T_{\beta}$. Using the numerical values chosen in Eq. (7) an example is given in Fig. 11a in the case $T=$ $T_{\alpha}+\frac{2}{3} T_{\beta}$. Let us now look at what happens for decreasing values of $T$. The use of problem (14) for the calculation of a periodic solution relies on the fact that the particle remains at rest at the point $\{\tilde{u}, 0\}$ up to the time when the perturbation of the loading is set to $P(t)=\varepsilon$ again. If $\tilde{u}<u^{-}$, the point $\{\tilde{u}, 0\}$ is not an equilibrium point, so that the reaction of the particle passing through this point can only jump to the other side of the cone and the particle starts sliding in the other direction. In other words, formula (15) no longer applies because problem (14) itself no longer applies. Looking at formula (15) we see that $x$ is a continuously decreasing function of $T$ in the interval $] T_{\alpha}, T_{\alpha}+T_{\beta}$ [, so that there exists in this interval a single value of $T$ depending on $\varepsilon$ for which the initial data $u_{0}$ is such that $u_{0}=u^{-}$. Let $T_{0}$ be this value connected to $\varepsilon$ by the following equation:

$$
\begin{aligned}
& T_{0}-T_{\alpha}=\frac{1}{\omega_{\beta}} \arccos \\
& \frac{u^{\varepsilon-}-u^{\varepsilon+}-\left[\frac{2 \omega_{\beta}^{2}}{\varepsilon}\left(u^{\varepsilon-}-u^{\varepsilon+}\right)+1\right]\left(u^{\varepsilon-}-u^{-}\right)}{2 u^{\varepsilon-}-u^{-}-u^{\varepsilon+}} .
\end{aligned}
$$

We thus have an explicit expression of the boudary $\Gamma_{12}$. We could easily modify problem (14) in order to take one more sliding phase into account, but one can check that this will not give a periodic solution of period $2 T$ starting with a zero velocity at time $t=0$ if $\tilde{u}<u^{-}$, that is if $T$ is smaller than the left boundary of Zone $\Omega_{1}$. The reason being that there does not remain enough time for a complete new sliding phase to occur up to an equilibrium point before $t=2 T$

We shall see that there nevertheless still exist periodic solutions, but they will not start with a zero velocity at the origin. This means that the left side of Zone $\Omega_{1}$ will be characterized by the occurence of a phase difference between the periodic loading and the response (see Fig. 11b).

\subsubsection{Zone $\Omega_{2}$ : periodic solutions on the left of $\Omega_{1}$ :}

As a consequence of Sect. 4.2.1, when $(T, \varepsilon)$ belongs to $\Omega_{2}$ we look for a periodic solution starting at time $t=0$ from a point $\left\{u_{0}, v_{0} \neq 0\right\}$. The trajectory can be qualitatively described as follows: the particle is sliding to the right (e.g. with a positive tangential velocity $v_{0}$ ) when $P(t)$ is set to $\varepsilon$, the particle goes on sliding to the right up to a stop, then the reaction jumps to the other side of the cone and the particle slides to the left. Then $P(t)$ is set to zero but the particle goes on sliding to the left up to a stop, the reaction then jumps to the other side of the cone and the particle slides to the right, but $P(t)$ is set to $\varepsilon$ again before the particle stops. The phase difference is qualitatively represented on Fig. 12.

Such a trajectory is the solution of the following system:

$$
\left\{\begin{array}{l}
\ddot{u}_{1}+\omega_{\alpha}^{2} u_{1}=F_{t}-\mu F_{n}+\varepsilon, t \in(0, \tilde{t}) \\
u_{1}(0)=u_{0}, \dot{u}_{1}(0)=v_{0}, \\
\tilde{t} \text { such that } \dot{u}_{1}(\tilde{t})=0, \\
\ddot{u}_{2}+\omega_{\beta}^{2} u_{2}=F_{t}+\mu F_{n}+\varepsilon, t \in(\tilde{t}, T) \\
u_{2}(\tilde{t})=u_{1}(\tilde{t}), \dot{u}_{2}(\tilde{t})=0, \\
\ddot{u}_{3}+\omega_{\beta}^{2} u_{3}=F_{t}+\mu F_{n}, t \in(T, \hat{t}) \\
u_{3}(T)=u_{2}(T), \dot{u}_{3}(T)=\dot{u}_{2}(T), \\
\hat{t} \text { such that } \dot{u}_{3}(\hat{t})=0, \\
\ddot{u}_{4}+\omega_{\alpha}^{2} u_{4}=F_{t}-\mu F_{n}, t \in(\hat{t}, 2 T) \\
u_{4}(\hat{t})=u_{3}(\hat{t}), \dot{u}_{4}(\hat{t})=0,
\end{array}\right.
$$

and the existence of a periodic solution of period $2 T$ is obtained by computing a solution to the following system:

$u_{4}(2 T)=u_{0}, \dot{u}_{4}(2 T)=v_{0}$.

From system (17) the periodicity condition (18) can be rewritten as an algebraic equation involving the unknowns $u_{0}$ and $v_{0}$ :
Fig. 11 The difference between periodic solutions on both sides of $\Gamma_{12}$ for $\varepsilon=1.5$.
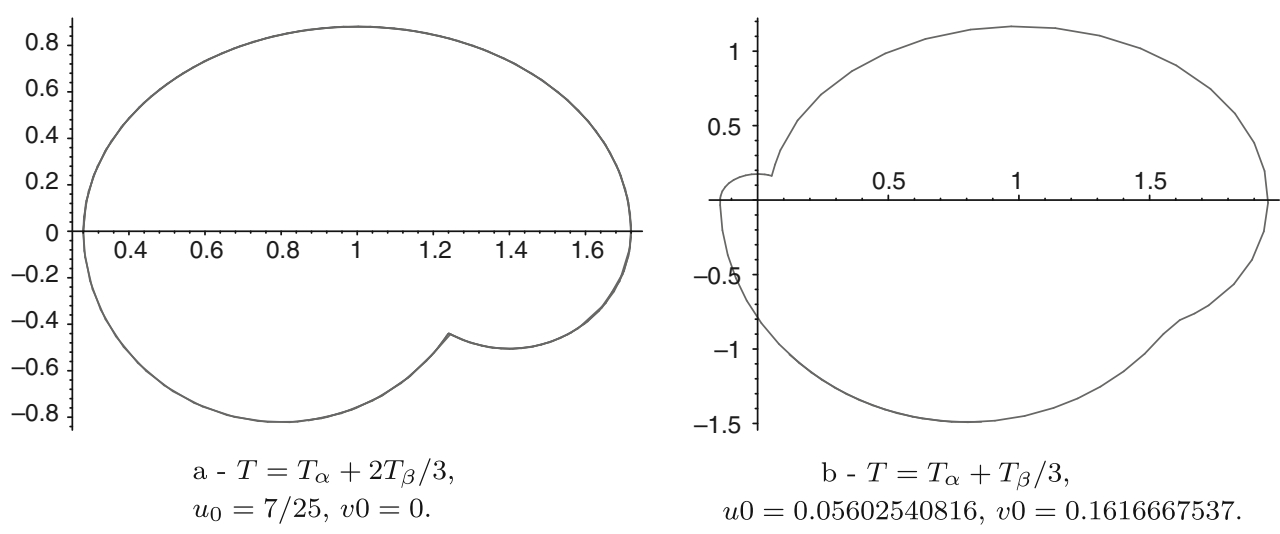


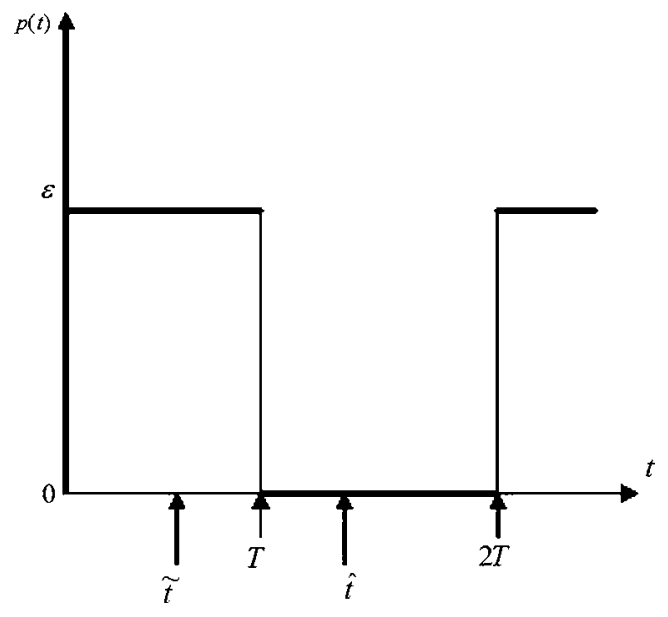

Fig. 12 The phase difference on the left of Zone $\Omega_{1}$

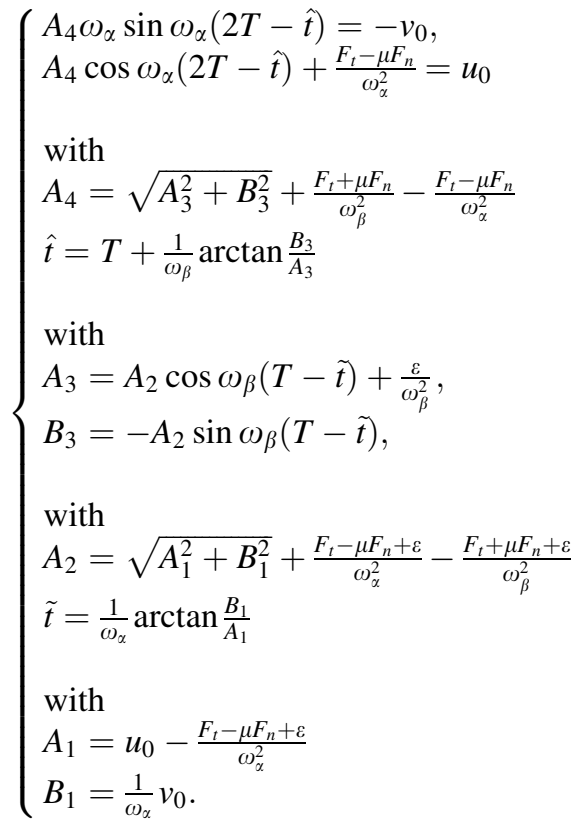

This intricate algebraic system of two equations with unknowns $\left(u_{0}, v_{0}\right)$ is solved using the software Maple. For the values given in Eq. (7) we obtain the initial data and we can then plot the periodic solution satisfying system (17) (see Fig. 11 (b)).

\subsubsection{Boundary $\Gamma_{23}$ : delimiting the zone where periodic solutions on the left of $\Omega_{1}$ exist}

The solution to problem (19) proves the existence of periodic solutions on the left of Zone $\Omega_{1}$. But these solutions hold only for $T$ sufficiently close to the value $T_{0}$ given by 16 . As a matter of fact, the solution to problem (17-19) for different values of $T$ shows that the amplitude of the periodic solution increases progressively as $T$ decreases in such a way that the maximum value $u_{\max }$ of the sliding tangential displacement is reached for a reaction closer and closer to the vertex of the cone. If the reaction of a solution of problem (17-19) goes through the vertex, then the solution does not fulfill the unilateral contact condition and is therefore no longer a solution to the initial problem. The following steps give the key for the calculation of the boundary for this occurence:

1. the solution to system (17) is such that $u_{\max }=u_{1}(\tilde{t})$;

2. it is immediately seen from problem (1) that the vertex of the cone, that is $R_{n}=0$, corresponds to a tangential displacement equal to $\frac{F_{n}}{W}$;

3. according to remark 2, problem (1) gives a linear relation between $u_{t}$ and $R_{n}$ for $u_{n} \equiv 0$;

4. consequently the algebraic system (19) can be seen as a map which associates the maximal value of $R_{n}$ with any pair $(T, \varepsilon)$ for which there exists a periodic solution of the type (17), (18), (19). Let $\mathcal{F}(T, \varepsilon)$ be this map.

The occurence of solutions loosing contact in the $\{T, \varepsilon\}$ plane is then given by the implicit solution $\varepsilon=\varepsilon(T)$ of the following equation:

$\mathcal{F}(T, \varepsilon)=0$,

so that Eq. (20) defines the boundary $\Gamma_{23}$.

4.2.4 Zone $\Omega_{5}: T_{\beta}<T<T_{\alpha}$

For decreasing values of $\mathrm{T}$, the solution to Eq. (20) decreases up to values of $\varepsilon$ very close to the limit of the range where there exist only equilibrium solutions. But in fact we observe that this curve is not tangent to the horizontal limit of stationary solutions. There remains a thin layer in which we shall obtain periodic solutions with a new kind of orbit. As suggested by numerical experiments periodic solutions in this layer satisfy the following system:

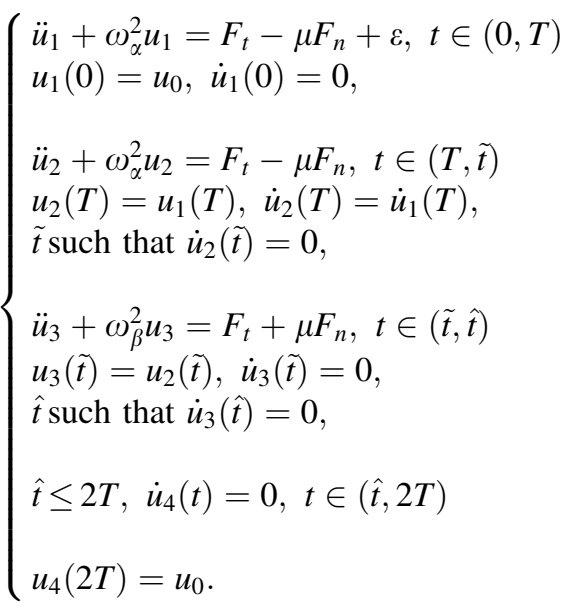

The periodicity condition reduces to $u_{3}(\hat{t})=u_{0}$, which leads to an algebraic system much simpler than (19) for the 
determination of the initial data $u_{0}$. The solution is represented on Fig. 13. It is clear from the conditions written in problem (21) that such solutions exist only if $T_{\beta}<T<T_{\alpha}$ Moreover, the initial data of this kind of solution is such that $u_{0}$ is strictly positive by construction. It is then natural to look for the border of Zone $\Omega_{5}$ by calculating the loss of positivity of the initial data in the range $T_{\beta}<T<T_{\alpha}$. This can be done in the same way as presented in Sect. 4.2 .3 by the study of the implicit solution of Eq. (20). But it is easily calculated that in $\Omega_{5}$ the maximum $u_{\max }$ of the sliding displacement never corresponds to a reaction at the vertex of the cone, so that, assuming the continuity of the solution with repect to $T$ and $\varepsilon$ there necessarily remains a very thin layer between $\Omega_{5}$ and $\Omega_{3}$ as we shall see in the next section.

\subsubsection{Zone $\Omega_{4}$ : periodic solutions for small $T$}

The above procedure can easily be adapted to prove that periodic solutions exist even for very high frequencies of the excitation. The solutions in Zones $\Omega_{2}$ and $\Omega_{5}$ suggest that Zone $\Omega_{4}$ may contain periodic solutions starting with a negative velocity. This means that system (17) should be changed into

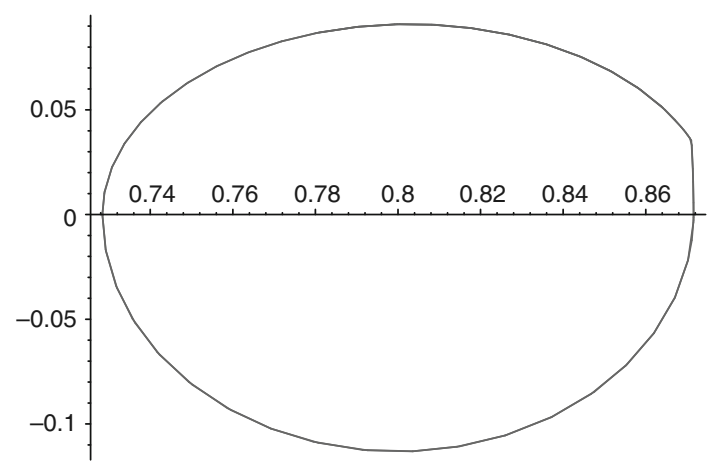

Fig. 13 A periodic solution in $\Omega_{5}$

$$
\left\{\begin{array}{l}
\ddot{u}_{1}+\omega_{\beta}^{2} u_{1}=F_{t}+\mu F_{n}+\varepsilon, t \in(0, \tilde{t}) \\
u_{1}(0)=u_{0}, \dot{u}_{1}(0)=v_{0}, \\
\tilde{t} \text { such that } \dot{u}_{1}(\tilde{t})=0, \\
\ddot{u}_{2}+\omega_{\alpha}^{2} u_{2}=F_{t}-\mu F_{n}+\varepsilon, t \in(\tilde{t}, T) \\
u_{2}(\tilde{t})=u_{1}(\tilde{t}), \dot{u}_{2}(\tilde{t})=0, \\
\ddot{u}_{3}+\omega_{\alpha}^{2} u_{3}=F_{t}-\mu F_{n}, t \in(T, \hat{t}) \\
u_{3}(T)=u_{2}(T), \dot{u}_{3}(T)=\dot{u}_{2}(T), \\
\hat{t} \operatorname{such} \text { that } \dot{u}_{3}(\hat{t})=0, \\
\ddot{u}_{4}+\omega_{\beta}^{2} u_{4}=F_{t}+\mu F_{n}, t \in(\hat{t}, 2 T) \\
u_{4}(\hat{t})=u_{3}(\hat{t}), \dot{u}_{4}(\hat{t})=0,
\end{array}\right.
$$

corresponding to trajectories represented on Fig. 14 in the $\left\{R_{t}, R_{n}\right\}$ plane. The periodicity condition is exactly the same requirement as Eq. (18), with an explicit form very close to (19). We then get initial data $\left(u_{0}, v_{0}\right)$ and system (22) gives the corresponding periodic solution in the same way as previously.

In fact solving system (22) through the calculation of initial data which belong to a periodic trajectory does not require $T$ to be very small, so that starting from very small $T$ one can increase $T$ progressively and obtain periodic solutions of larger and larger amplitude for the same value of $\varepsilon$ chosen sufficiently large. Figure 15 contains different periodic solutions for increasing values of $T$ but represented at the same scale. The corresponding values of the initial data given in the caption are computed through the above scheme. Figure 15 brings us to formulate the following remark.

\section{Remark 4}

- These periodic solutions are all homeomorphic to an ellipse,

- for a given $\varepsilon$ the amplitude of the sliding oscillation decreases as $T$ decreases,
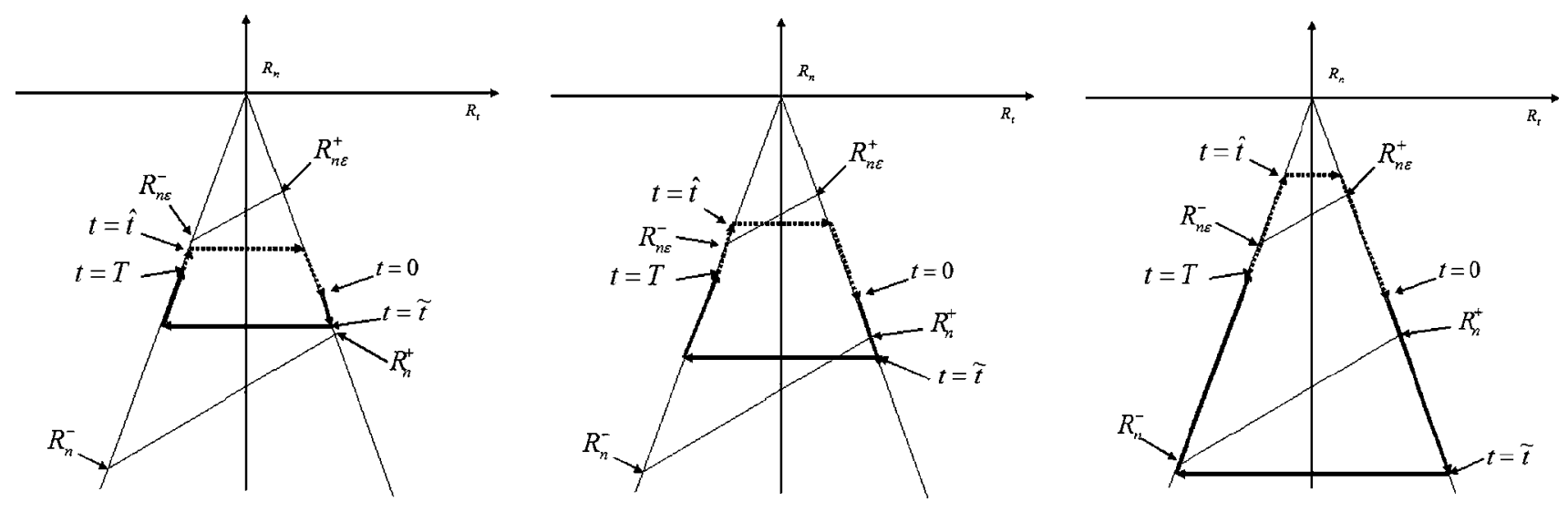

Fig. 14 Periodic solutions for small values of $T$ in the $\left\{R_{t}, R_{n}\right\}$ plane 

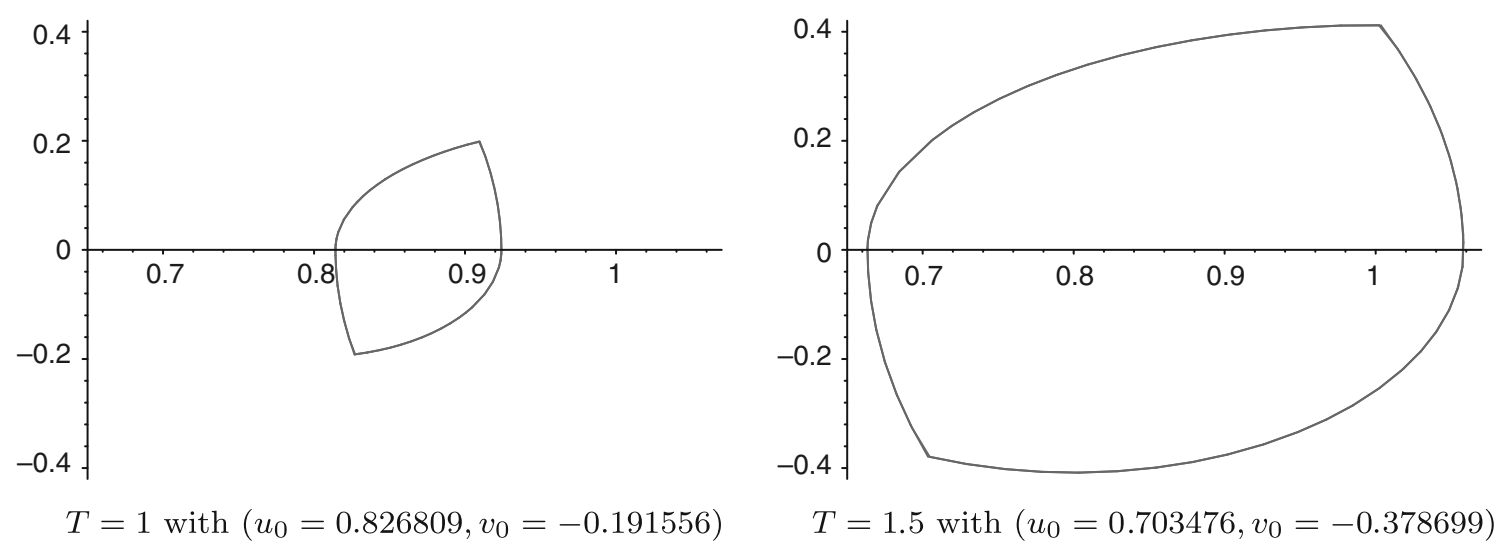

Fig. 15 Periodic solutions in the phase space $\left(u_{t}, \dot{u}_{t}\right)$ when $\varepsilon=1.5$ and $\left(u_{0}, v_{0}\right)$ are obtained by (22)

- for a given $T$ the amplitude of the sliding oscillation decreases as $\varepsilon$ increases,

- the phase difference increases as $T$ decreases. Moreover, the phase difference is always larger than in Zone $\Omega_{2}$.

\section{The transition for loosing contact}

\subsection{Exploring the upper boundary}

Section 4 started to partition the $\{T, \varepsilon\}$ plane into zones where different kinds of periodic solutions have been exhibited. These solutions satisfy strictly the Coulomb friction law but they are bilateral by which we mean that they satisfy the unilateral contact conditions $U_{n} \leq 0$, $R_{n} \leq 0, \quad U_{n} R_{n}=0$ by satisfying $U_{n}=0, \quad R_{n}<0$. We already know that the horizontal line $\varepsilon=\frac{2 \mu \mathcal{A}}{K_{t}+\mu W}$ for any $T$ is the lower limit of the existence of periodic solutions, which means in particular that this line is the lower boundary of all the zones of existence of any type of periodic solutions described up to now. Concerning the upper boundary of these zones we mentioned the possibility for a particular periodic solution to loose contact, this was the transition from $\Omega_{2}$ to $\Omega_{3}$. As the last step of the present work, we shall determine this upper boundary, defined by the occurence of one point loosing contact during a period, on the whole $T$ axis.

\subsubsection{Boundary $\Gamma_{34}$ : from very small periods to $T_{\beta}$}

We are now looking for the transition between sliding and loosing contact. Let $\widetilde{\mathcal{F}}(T, \varepsilon)$ be the maximal value of the normal component of the reaction corresponding to the periodic solution for a given $(T, \varepsilon)$. Then at the transition for loosing contact, $T$ and $\varepsilon$ satisfy
$\widetilde{\mathcal{F}}(T, \varepsilon)=0$.

In Sect. 4.2 .3 the mapping $\widetilde{\mathcal{F}}$ was defined by Eq. (20) built from problem (17 - 18 - 19). Starting here from problem (22), the transition for loosing contact is given in the $\{T, \varepsilon\}$ plane by the implicit solution of Eq. (23) from $T$ close to zero to a neighbourhood of $T_{\beta}$.

\subsubsection{From $T_{\beta}$ to $T_{\alpha}$}

Zone $\Omega_{5}$ of Fig. 10b has been defined for $\left.T \in\right] T_{\beta}, T_{\alpha}[$. In this range, there exists a periodic solution of the type represented on Fig. 13. We can check that everywhere in Zone $\Omega_{5}$, these periodic solutions are such that

$\widetilde{\mathcal{F}}(T, \varepsilon)<0$

which means that the periodic solutions in Zone $\Omega_{5}$ never reach the transition for loosing contact. This implies that there must exist a transition between Zone $\Omega_{5}$ and Zone $\Omega_{3}$ where another type of solution would loose contact when $\varepsilon$ increases, at least for $T$ in some subinterval of $] T_{\beta}, T_{\alpha}[$. Periodic solutions in a thin layer can actually be obtained from the following system (25) which continues Zone $\Omega_{5}$ after the loss of positivity of the initial position ${ }^{1}$.

\footnotetext{
${ }^{1}$ Let us compare problems (21) and (25). It appears that proving that (21) has a solution everywhere in zone $\Omega_{5}$ amounts to proving that problem (25) has a solution such that $u_{4}$ is constant everywhere in a nonzero measure subset of the $\{T, \varepsilon\}$ plane, which could have been missed by a direct study of problem (25), and which is a result interesting in itself. Moreover distinguishing Zones $\Omega_{5}$ and $\Omega_{6}$ seems easier for an intuitive introduction to the partition of the plane. Nevertheless, the distinction between these two zones would not be necessary if we were dealing only with the transition to the loss of contact.
} 


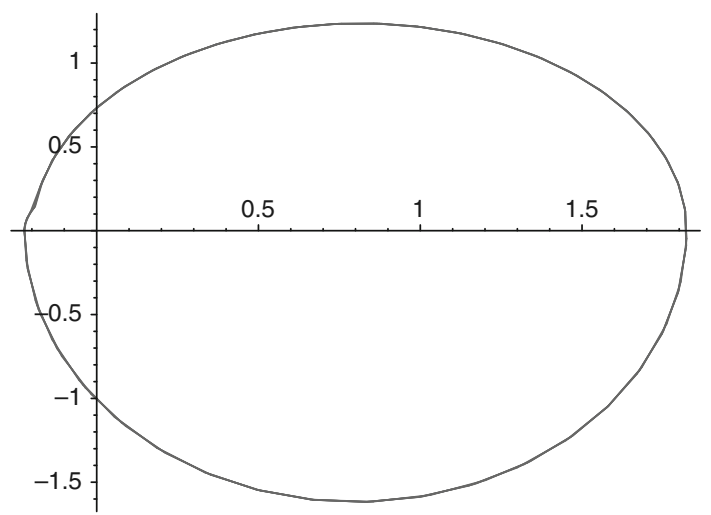

Fig. 16 A periodic solution in zone $\Omega_{6}$

$$
\left\{\begin{array}{l}
\ddot{u}_{1}+\omega_{\alpha}^{2} u_{1}=F_{t}-\mu F_{n}+\varepsilon, t \in(0, T) \\
u_{1}(0)=u_{0}, \dot{u}_{1}(0)=v_{0}, \\
\ddot{u}_{2}+\omega_{\alpha}^{2} u_{2}=F_{t}-\mu F_{n}, t \in(T, \tilde{t}) \\
u_{2}(T)=u_{1}(T), \dot{u}_{2}(T)=\dot{u}_{1}(T), \\
\tilde{t} \text { such that } \dot{u}_{2}(\tilde{t})=0, \\
\ddot{u}_{3}+\omega_{\beta}^{2} u_{3}=F_{t}+\mu F_{n}, t \in\left(\tilde{t}, \tilde{t}+T_{\beta}\right), \\
u_{3}(\tilde{t})=u_{2}(\tilde{t}), \dot{u}_{3}(\tilde{t})=0, \\
\ddot{u}_{4}+\omega_{\alpha}^{2} u_{4}=F_{t}-\mu F_{n}, t \in\left(\tilde{t}+T_{\beta}, 2 T\right) \\
u_{4}\left(\tilde{t}+T_{\beta}\right)=u_{3}\left(\tilde{t}+T_{\beta}\right), \dot{u}_{4}\left(\tilde{t}+T_{\beta}\right)=0,
\end{array}\right.
$$

together with the periodicity condition

$u_{4}(2 T)=u_{0}, \dot{u}_{4}(2 T)=v_{0}$.

Let $R_{\text {max }}$ be the maximal value of the normal component of the reaction. Then using the solution of problem $(25,26)$ the equality $R_{\text {max }}=0$ is reached for some values of $T$ and $\varepsilon$ which are again obtained as the implicit solution of Eq. (23). This gives Zone $\Omega_{6}$ of Fig. 10 and the periodic solutions are represented on Fig. 16.

\subsubsection{From $T_{\alpha}$ to $T_{\alpha}+T_{\beta}$}

In Sect. 4 we gave the transition between Zone $\Omega_{1}$ and Zone $\Omega_{2}$. Since the periodic solutions in Zone $\Omega_{1}$ are given by an explicit formula, we obtain analytically the function which gives the boundary $\Gamma_{12}{ }^{2}$, and the domain of this function on the $T$ axis appears to be divided into two subintervals according to whether the first loss of validity of the periodic solutions in Zone $\Omega_{1}$ is a loss of contact or a phase difference. In the left subinterval we obtained the transition already described by the occurrence of a phase difference, but for larger values of $T$ we get that the loss of contact occurs first in a range corresponding to large values

\footnotetext{
${ }^{2}$ In fact in this range the complete calculations are obviously also carried out using Maple, but here from explicit formula.
}

of $\varepsilon$. In other words this means that when the dynamical problem is built with Eqs. $(14,15)$, equation (23), which is here simpler than in general because more explicit, has a solution $\varepsilon=\varepsilon(T)$ only in a subinterval of $] T_{\alpha}, T_{\alpha}+T_{\beta}[$.

\subsubsection{From $T_{\alpha}+T_{\beta}$ to very long periods}

Proposition 3 in Sect. 4.1 gave the existence of infinitely many periodic solutions everywhere in the open range ]$T_{\alpha}+T_{\beta},+\infty[\times] \frac{2 \mu \mathcal{A}}{K_{t}+\mu W}, \frac{4 \mu \mathcal{A}}{K_{t}+3 \mu W}[$ and a single periodic solution on the upper boundary of this range which is the line $] T_{\alpha}+T_{\beta},+\infty\left[\times\left\{\frac{4 \mu \mathcal{A}}{K_{t}+3 \mu W}\right\}\right.$, and we can easily check that condition (24) is satisfied all along this line. This means that none of the periodic solutions given by Proposition 3 are on the point of loosing contact. Again, this suggests that there might exist another zone where another type of sliding periodic solution exists up to the loss of contact $^{3}$. Since the upper boundary $\varepsilon=\frac{4 \mu \mathcal{A}}{K_{t}+3 \mu W}$ is exactly the coalescence of the two loops of periodic solutions of period $4 T$ into the single loop of a solution of period $2 T$, we could look for these new periodic solutions again as solutions with two loops. Such solutions satisfy the following system:

$$
\left\{\begin{array}{l}
\ddot{u}_{1}+\omega_{\alpha}^{2} u_{1}=F_{t}+\varepsilon-\mu F_{n}+\varepsilon, t \in\left(0, T_{\alpha}\right) \\
u_{1}(0)=u_{0}, \dot{u}_{1}(0)=0, \\
\ddot{u}_{2}+\omega_{\beta}^{2} u_{2}=F_{t}+\varepsilon+\mu F_{n}, t \in\left(T_{\alpha}, T_{\alpha}+T_{\beta}\right) \\
u_{2}\left(T_{\alpha}\right)=u_{1}\left(T_{\alpha}\right), \dot{u}_{2}\left(T_{\alpha}\right)=0, \\
\ddot{u}_{3}+\omega_{\alpha}^{2} u_{3}=F_{t}+\varepsilon-\mu F_{n}, t \in\left(T_{\alpha}+T_{\beta}, T\right) \\
u_{3}\left(T_{\alpha}+T_{\beta}\right)=u_{2}\left(T_{\alpha}+T_{\beta}\right), \dot{u}_{3}\left(T_{\alpha}+T_{\beta}\right)=0, \\
\ddot{u}_{4}+\omega_{\alpha}^{2} u_{4}=F_{t}-\mu F_{n}, t \in(T, \tilde{t}) \\
u_{4}(T)=u_{3}(T), \dot{u}_{4}(T)=\dot{u}_{3}(T), \\
\tilde{t} \operatorname{such} \text { that } \dot{u}_{4}(\tilde{t})=0, \\
\ddot{u}_{5}+\omega_{\beta}^{2} u_{5}=F_{t}+\mu F_{n}, t \in\left(\tilde{t}, \tilde{t}+T_{\beta}\right) \\
u_{5}(\tilde{t})=u_{4}(\tilde{t}), \dot{u}_{5}(\tilde{t})=0, \\
u_{6}(t)=u_{5}\left(\tilde{t}+T_{\beta}\right), \dot{u}_{6}(t)=0, t \in\left(\tilde{t}+T_{\beta}, 2 T\right) .
\end{array}\right.
$$

The periodicity condition is now $u_{6}(2 T)=u_{0}$, which again leads to an algebraic system of the same type as (19) for the determination of the initial data $u_{0}$. Periodic solutions in

\footnotetext{
3 A difficulty has already been encountered when studying the transition from Zone $\Omega_{5}$ to Zone $\Omega_{6}$ : since we don't know a lot about the qualitative behaviour, another guess could be that the loss of contact arises only through non periodic solutions so that the line ]$T_{\alpha}+T_{\beta},+\infty\left[\times\left\{\frac{4 \mu \mathcal{A}}{K_{t}+3 \mu W}\right\}\right.$ would be the boundary for the loss of periodicity, instead of a transition between different types of periodic solutions. The answer is given if problem (27) has a solution.
} 


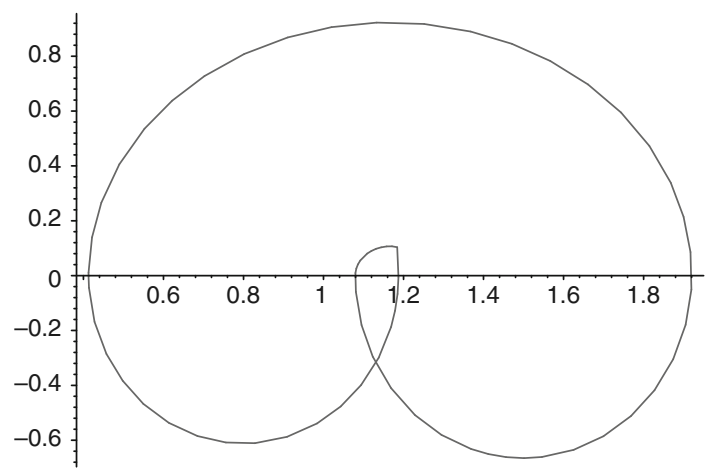

Fig. 17 Sliding periodic solution for large $\varepsilon$ and $T>T_{\alpha}+T_{\beta}$

this range have orbits of the type represented on Fig. 17, and the condition for loosing contact is obtained through the implicit solution of Eq. (23).

\subsection{Comments on the global results}

The qualitative behaviour of the periodic solutions of problem (1) is summarized in Fig. $18^{4}$. Let us comment this global result, paying a particular attention to the continuous curve which corresponds to the loss of contact. Let $\varepsilon(T)$ be this curve.

- Due to dissipation, excitations of any frequency lead to a strictly stuck equilibrium as long as their amplitude is smaller than $\frac{2 \mu \mathcal{A}}{K_{t}+\mu W}$. So that Fig. 18 represents only the part of the $\{T, \varepsilon\}$ plane above the line $\varepsilon=\frac{2 \mu \mathcal{A}}{K_{t}+\mu W}$.

- The minimum value of $\varepsilon(T)$ is very close to the loss of existence of equilibrium states. This means that this minimum could be interpreted as a resonance.

- Conversely, when $T \longrightarrow 0$ there exist periodic solutions strictly in contact for values of $\varepsilon$ which are large compared to the largest value of $\varepsilon$ for which there exists an equilibrium under static loading. Using the values given in (7), the latter is $\varepsilon=3$ while $\varepsilon(T) \simeq 6$ for $T$ close to zero. The curve $\varepsilon(T)$ is far from reaching this value in any other zone.

- The intricate behaviour of the periodic solutions is localized in a very small and thin range close to the minimum of $\varepsilon(T)$.

- We found only one zone where there exist more than one periodic solution for each given values of $T$ and $\varepsilon$.

\footnotetext{
${ }^{4}$ It is interesting to observe that, while giving the transition between Zone $\Omega_{1}$ and Zone $\Omega_{2}$ only amounts to plotting the graph of an explicit function, calculating all the other curves of Fig. 18 requires an implicit computation that can be very time consuming.
}

This zone is a horizontal strip which goes from $T_{\alpha}+T_{\beta}$ to $+\infty$. Everywhere in this strip there exist infinitely many periodic solutions. All these solutions are such that $R_{n}$ is strictly negative at any time so that they all remain strictly in contact.

\section{Concluding remarks}

After an investigation of the equilibrium states and of the qualitative properties of the trajectories going to equilibrium, we delimit in this work the zone where periodic solutions remain in contact, and we explore the shapes of the periodic orbits. This study could be taken as an example of the possible qualitative behaviour of a simple discrete system with unilateral contact and Coulomb friction.

A complete investigation of the possible behaviours would also require an analysis of the dependence with respect to other parameters of the system.

- Different choices of the stiffness parameters and friction coefficient would change the coupling between the degrees of freedom, and change the set of equilibrium states at the origin of this dynamical study (see [5]).

- The excitation has been reduced in the present study to a periodic perturbation $P(t)$ of the tangential component of the load. If the perturbation concerns strictly the normal component then all the trajectories lead to an equilibrium. If $P(t)$ concerns both components then periodic solutions appear for larger amplitudes of the perturbation, however the qualitative features of Fig. 18 seem to remain.

- Relatively complete analytical calculations were possible due to the particular choice of the excitation and a thorough investigation has thus been obtained. The behaviour in the case of a general periodic excitation remains to be explored.

The periodic solutions obtained are all such that the normal displacement remains equal to zero for all time, although problem (1) allows unilateral contact. The normal component of the reaction appears only through the coupling in the mechanical system and through the Coulomb friction law. Solutions where the motion involves periods of loss of contact are subject to impacts. Taking impacts into account will first increase the size of the system since $u_{n}$ becomes unknown, which rules out the use of Eqs. (3) and (4), and above all will add an extra nonsmoothness. It is known that the occurence of chaos or the persistence of periodic solutions in systems with impacts have a vast 


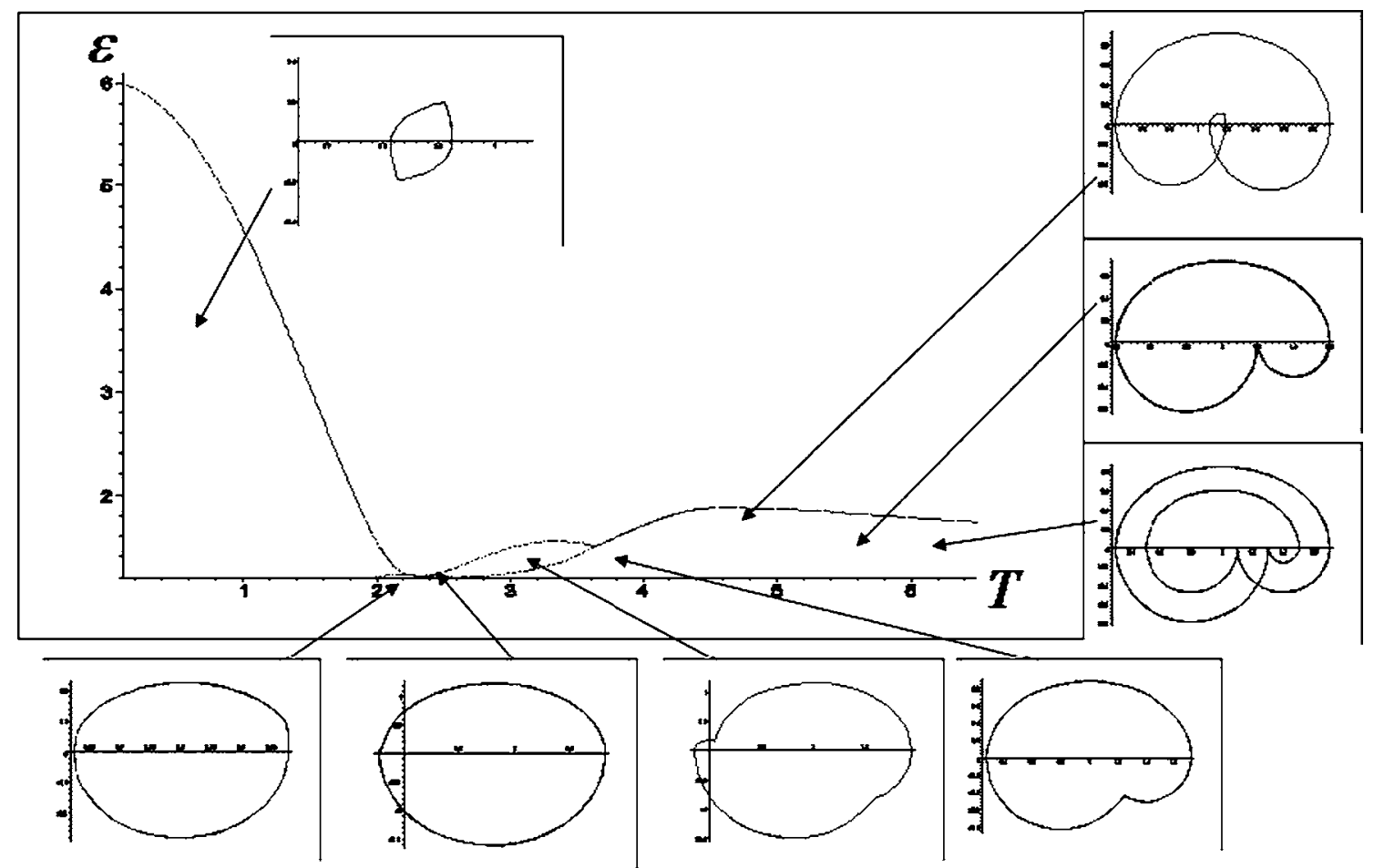

Fig. 18 The global behaviour in the period-amplitude plane

number of engineering applications. Our previous careful consideration of friction together with schemes able to deal very rigorously with impacts (see [8]) will be of the utmost importance in this future study. We are at present working on these lines.

\section{References}

1. Alart P, Curnier A (1986) Contact discret avec frottement: unicité de la solution, convergence de l'algorithme. Publications du Laboratoire de Mécanique Appliquée. Ecole Polytechnique Fédérale de Lausanne

2. Ballard P, Léger A, Pratt E (2006) Stability of discrete systems involving shocks and friction. In: Wriggers $P$, Nackenhorst U (eds) Analysis and simulation of contact problems. Lecture notes in applied and computational mechanics. Springer, Berlin, pp 343-350

3. Basseville S, Léger A (2006) Stability of equilibrium states in a simple system with unilateral contact and Coulomb friction. Arch Appl Mech 76(7/8):403-428

4. Ballard P, Basseville S (2005) Existence and uniqueness for dynamical unilateral contact with Coulomb friction: a model problem. Math Model Num Anal 39(1):57-77
5. Basseville S, Léger A, Pratt E (2003) Investigation of the equilibrium states and their stability for a simple model with unilateral contact and Coulomb friction. Arch Appl Mech 73:409-420

6. Brezis H (1973) Operateurs maximaux monotones et semigroupes de contraction dans les espaces de Hilbert. North Holland, Amsterdam

7. Geymonat G, Léger A (1993) Role de l'expérimentation numérique dans les problèmes non-linéaires à plusieurs paramètres. Matapli 35:35-51

8. Jean M (1999) The nonsmooth contact dynamics method. Comput Methods Appl Mech Eng 177:235-257

9. Klarbring A (1990) Examples of non-uniqueness and non-existence of solutions to quasistatic contact problems with friction. Ing Arch 60:529-541

10. Martins JAC, Monteiro Marques MDP, Gastaldi F (1994) On an example of non-existence of solution to a quasistatic frictional contact problem. Eur J Mech A Solids 13(1):113-133

11. Moreau JJ (1988) Unilateral contact and dry friction in finite freedom dynamics. In: Moreau JJ, Panagiotopoulos PD (eds) Nonsmooth mechanics and applications. CISM courses and lectures 302. Springer, Vienne

12. Pratt E, Léger A, Jean M (2008) Critical oscillations of massspring systems due to nonsmooth friction. Arch Appl Mech 78:89-104

13. Pratt E, Léger A, Jean M (2010) About a stability conjecture concerning unilateral contact with friction. Nonlinear Dyn 59:73-94 